\title{
Thom Isotopy Theorem for Nonproper Maps and Computation of Sets of Stratified Generalized Critical Values
}

\author{
Sĩ Tiệp Đinh ${ }^{1,2} \cdot$ Zbigniew Jelonek $^{1}$
}

Received: 10 May 2018 / Revised: 22 February 2019 / Accepted: 28 March 2019 / Published online: 15 April 2019 (c) The Author(s) 2019

\begin{abstract}
Let $X \subset \mathbb{C}^{n}$ be an affine variety and $f: X \rightarrow \mathbb{C}^{m}$ be the restriction to $X$ of a polynomial map $\mathbb{C}^{n} \rightarrow \mathbb{C}^{m}$. We construct an affine Whitney stratification of $X$. The set $K(f)$ of stratified generalized critical values of $f$ can also be computed. We show that $K(f)$ is a nowhere dense subset of $\mathbb{C}^{m}$ which contains the set $B(f)$ of bifurcation values of $f$ by proving a version of the Thom isotopy lemma for nonproper polynomial maps on singular varieties.
\end{abstract}

Keywords Isotopy lemma - Affine varieties · Nonproper polynomial mapping · Local trivial fibration

Mathematics Subject Classification Primary 32B20; Secondary 14PXX

\section{Introduction}

Ehresmann's fibration theorem [3] states that a proper smooth surjective submersion $f: X \rightarrow N$ between smooth manifolds is a locally trivial fibration. With some extra assumptions, this result has been considered in different contexts.

Firstly, if we remove the assumption of properness or submersiveness, in general, Ehresmann's fibration theorem does not hold, since $f$ might have "local singularities" or "singularities at infinity." The set of points in $N$ where $f$ fails to be trivial, denoted by

\section{Editor in Charge: Kenneth Clarkson}

Sĩ Tiệp Đinh

sdinh@impan.pl; dstiep@math.ac.vn

Zbigniew Jelonek

najelone@cyf-kr.edu.pl

1 Institute of Mathematics, Polish Academy of Sciences, Śniadeckich 8, 00-656 Warsaw, Poland

2 Institute of Mathematics, VAST, 18, Hoang Quoc Viet Road, Cau Giay District, Hanoi 10307, Vietnam 
$B(f)$, is called the bifurcation set of $f$, which is the union of the set $K_{0}(f)$ of critical values and the set $B_{\infty}(f)$ of bifurcation values at infinity of $f$. To date, characterizing $B_{\infty}(f)$ remains an open problem. In general, a larger (but easier to describe) set is used, viz. the set of asymptotic critical values of $f$ (see Definition 3.1), denoted by $K_{\infty}(f)$, to control $B_{\infty}(f)$. The set $K_{\infty}(f)$ is always a nowhere dense subset of $\mathbb{C}^{m}$ and is a good approximation of the set $B_{\infty}(f)$. For a dominant map $f: X \rightarrow \mathbb{C}^{m}$ on a smooth complex affine variety $X$, the computation of $K_{\infty}(f)$, and hence of the set of generalized critical values, $K(f):=K_{0}(f) \cup K_{\infty}(f)$, is given in [8-10].

Now, if $X$ is singular, one must partition it into disjoint smooth manifolds and then apply Ehresmann's fibration theorem on each part. However, if we do not require any extra assumptions, then the trivialization on the parts may not match. This obstacle can be overcome by introducing the Whitney conditions [21,22]. Indeed, if $f$ is proper and $X$ admits a Whitney stratification, then $f$ is locally trivial if it is a submersion on strata $[13,18,20]$. Moreover, if $f$ is nonproper and nonsubmersive, we can also define the bifurcation set of $f$ such that $f$ is locally trivial outside $B(f)$. However, to date, to the best of the authors' knowledge, no connection between $B(f)$ and the set of stratified generalized critical values of $f$, defined by $K(f, \mathcal{S}):=$ $\bigcup_{X_{\alpha} \in \mathcal{S}} K\left(f, X_{\alpha}\right)$, for a Whitney stratification $\mathcal{S}$ of $X$, has been established. Here $K\left(f, X_{\alpha}\right)=K_{0}\left(f, X_{\alpha}\right) \cup K_{\infty}\left(f, X_{\alpha}\right)$, where $K_{0}\left(f, X_{\alpha}\right)$ is the closure of the set of critical values of $f_{\mid X_{\alpha}}$ and $K_{\infty}\left(f, X_{\alpha}\right)=\left\{y \in \mathbb{C}^{m}\right.$ : there is a sequence $x^{k} \rightarrow$ $\infty, x^{k} \in X_{\alpha}$ such that $\left\|x^{k}\right\| v\left(\mathrm{~d}_{x^{k}}\left(\left.f\right|_{X_{\alpha}}\right)\right) \rightarrow 0$ and $\left.f\left(x^{k}\right) \rightarrow y\right\}$ (v denotes the Rabier function, see Sect. 5).

Let $X \subset \mathbb{C}^{n}$ be a singular algebraic set of dimension $n-r$ with $I(X)=$ $\left(g_{1}, \ldots, g_{\omega}\right)$, and let $f:=\left(f_{1}, \ldots, f_{m}\right): X \rightarrow \mathbb{C}^{m}$ be a polynomial dominant map, i.e., $\overline{f(X)}=\mathbb{C}^{m}$. Now, restricting ourselves to the cases of dominant polynomial maps on singular affine varieties, the main goals of this paper are the following:

- Construct an affine Whitney stratification $\mathcal{S}$ of $X$.

- Establish some version of the Thom isotopy lemma for $f$ which yields the inclusion $B(f) \subset K(f, \mathcal{S})$.

- Calculate the set $K(f, \mathcal{S})$ of stratified generalized critical values of $f$.

The remainder of this manuscript is organized as follows: In Sect. 2, we recall the definitions of Whitney regularity and Whitney stratification, then construct an affine stratification from a filtration of $X$ by means of some hypersurfaces, and refine it to obtain an affine Whitney stratification. Some versions of the Thom isotopy lemma for nonproper polynomial maps (Theorem 3.4 and Corollary 3.11) are given in Sect. 3. We then compute the set of stratified generalized critical values of $f$, which contains the bifurcation values of $f$, where $f:=\left(f_{1}, \ldots, f_{m}\right): X \rightarrow \mathbb{C}^{m}$ is a polynomial dominant map, in the final Sects. 4 and 5.

For the remainder of the paper, the differential of $f$ at a point $x$ is identified with its (row) matrix, so we write $\mathrm{d}_{x} f=\left(\frac{\partial f}{\partial x_{1}}(x), \ldots, \frac{\partial f}{\partial x_{n}}(x)\right)$. Let

$$
\nabla f(x):=\left[\begin{array}{c}
\overline{\frac{\partial f}{\partial x_{1}}(x)} \\
\vdots \\
\overline{\frac{\partial f}{\partial x_{n}}(x)}
\end{array}\right],
$$


the Hermitian transpose of $\mathrm{d}_{x} f$. For $v, w \in \mathbb{C}^{n}$, denote by $\langle v, w\rangle=\sum_{i=1}^{n} \bar{v}_{i} w_{i}$ the Hermitian product, and let $v \cdot w=\sum_{i=1}^{n} v_{i} w_{i}$. For a set $A \subset \mathbb{C}^{n}$, let $\bar{A}$ and $\bar{A} \mathcal{Z}$ denote respectively the topological closure and the Zariski closure of $A$. For an algebraic variety $X$, the singular part and the regular part of $X$ are denoted respectively by $\operatorname{sing}(X)$ and $\operatorname{reg}(X)$.

\section{Affine Whitney Stratifications}

\subsection{Preliminaries}

For any two different points $x, y \in \mathbb{C}^{n}$, define the secant $\overline{x y}$ to be the line passing through the origin which is parallel to the line through $x$ and $y$.

A stratification $\mathcal{S}$ of $X$ is a decomposition of $X$ into a locally finite disjoint union $X=\bigsqcup_{\alpha \in I} X_{\alpha}$ of nonempty, nonsingular, connected, locally closed subvarieties, called strata, such that the boundary $\partial X_{\alpha}:=\bar{X}_{\alpha} \backslash X_{\alpha}$ of any stratum $X_{\alpha}$ is a union of strata. If, in addition, for each $\alpha$, the closure $\bar{X}_{\alpha}$ and the boundary $\partial X_{\alpha}$ are affine varieties, then we call $\mathcal{S}$ an affine stratification. It is obvious that any affine stratification is finite.

For linear subspaces $F, G \subseteq \mathbb{C}^{n}$, let

$$
\delta(F, G):=\sup _{\substack{x \in F \\\|x\|=1}} \operatorname{dist}(x, G),
$$

where $\operatorname{dist}(x, G)$ is the Hermitian distance.

Let $\left(X_{\alpha}, X_{\beta}\right)$ be a pair of strata of $\mathcal{S}$ such that $X_{\beta} \subset \bar{X}_{\alpha}$ and let $x \in X_{\beta}$. We recall some regularity conditions:

(a) The pair $\left(X_{\alpha}, X_{\beta}\right)$ is said to be Whitney (a) regular at $x \in X_{\beta}$ if it satisfies the following Whitney condition (a) at $x$ : if $x^{k} \in X_{\alpha}$ is any sequence such that $x^{k} \rightarrow x$ and $T_{x^{k}} X_{\alpha} \rightarrow T$, then $T \supset T_{x} X_{\beta}$.

(w) The pair $\left(X_{\alpha}, X_{\beta}\right)$ is said to be (w) regular at $x \in X_{\beta}$ (or strictly Whitney (a) regular at $x$ with exponent 1 ) if it satisfies the following condition (w) at $x$ : there exist a neighborhood $U$ of $x$ in $\mathbb{C}^{n}$ and a constant $c>0$ such that, for any $y \in X_{\alpha} \cap U$ and $x^{\prime} \in X_{\beta} \cap U$, we have $\delta\left(T_{x^{\prime}} X_{\beta}, T_{y} X_{\alpha}\right) \leqslant c\left\|y-x^{\prime}\right\|$.

(b) The pair $\left(X_{\alpha}, X_{\beta}\right)$ is said to be Whitney regular at $x \in X_{\beta}$ if it satisfies the following Whitney condition (b) at $x$ : for any sequences $x^{k} \in X_{\alpha}$ and $y^{k} \in X_{\beta}$, $y^{k} \neq x^{k}$, such that $x^{k} \rightarrow x, y^{k} \rightarrow x, T_{x^{k}} X_{\alpha} \rightarrow T$, and $\overline{x^{k} y^{k}}$ converges to a line $\ell$ in the projective space $\mathbb{P}^{n-1}$, we have $\ell \subset T$.

We say that the pair $\left(X_{\alpha}, X_{\beta}\right)$ is Whitney (a) regular (resp. Whitney regular) if it is Whitney (a) regular (resp. Whitney regular) at every point of $X_{\beta}$. We say that $\mathcal{S}$ is a Whitney (a) stratification (resp. a Whitney stratification) if any pair of strata ( $X_{\alpha}, X_{\beta}$ ) of $\mathcal{S}$ with $X_{\beta} \subset \bar{X}_{\alpha}$ is Whitney (a) regular (resp. Whitney regular). It is well known that Whitney regularity implies Whitney (a) regularity $[21,22]$. Moreover, in light of [17, V.1.2], the Whitney condition (b) is equivalent to the condition (w) for 
the category of complex analytic sets, so to check Whitney regularity, we can verify either condition (w) or condition (b).

For the purpose of this paper, we also need the following notion of Whitney (resp. Whitney (a)) regularity along a stratum: Let $X_{\beta}$ be a stratum of $\mathcal{S}$ and let $x \in X_{\beta}$. We say that $X_{\beta}$ is Whitney regular (resp. Whitney (a) regular) at $x$ if, for any stratum $X_{\alpha}$ such that $X_{\beta} \subset \bar{X}_{\alpha}$, the pair $\left(X_{\alpha}, X_{\beta}\right)$ is Whitney (resp. Whitney (a)) regular at $x$. The stratum $X_{\beta}$ is Whitney regular (resp. Whitney (a) regular) if it is Whitney (resp. Whitney (a)) regular at every point of $X_{\beta}$. It is clear that $\mathcal{S}$ is a Whitney (resp. a Whitney (a)) stratification if and only if each stratum of $\mathcal{S}$ is Whitney (resp. Whitney (a)) regular.

\subsection{Construction of Affine Stratifications}

Let us, first of all, fix an affine stratification of $X$ whose construction is based on the following proposition:

Proposition 2.1 Let $X \subset \mathbb{C}^{n}$ be an affine subvariety of pure codimension $r$. Assume that $I(X)=\left(g_{1}, \ldots, g_{\omega}\right)$, where $\operatorname{deg} g_{i} \leq D$. Let $W$ be an affine subvariety of positive codimension in $X$ with $I(W)=\left(g_{1}, \ldots, g_{\omega}, u_{1}, \ldots, u_{\tau}\right)$, where $u_{i} \notin I(X)$ and $\operatorname{deg} u_{i} \leqslant D^{\prime}$. Then there exists a polynomial $p_{X, W}$ on $\mathbb{C}^{n}$ of degree less than or equal to $r(D-1)+D^{\prime}$ such that $W \subseteq V\left(p_{X, W}\right):=\left\{x \in \mathbb{C}^{n}: p_{X, W}(x)=0\right\}$ and $X \backslash V\left(p_{X, W}\right)$ is a smooth, dense subset of $X$. Moreover, the polynomial $p_{X, W}$ can be constructed effectively.

Proof Let $X=\bigcup_{i=1}^{m} Y_{i}$, where $Y_{i}$ are irreducible (hence $r$-codimensional) components of $X$. Take sufficiently generic (random) rational numbers $\alpha_{i j} \in \mathbb{Q}, i=1, \ldots, r$, $j=1, \ldots, \omega$, and set

$$
G_{i}=\sum_{j=1}^{\omega} \alpha_{i j} g_{j}, \quad i=1, \ldots, r
$$

Here and in the following, to obtain a generic number, it is sufficient to take a random rational number and verify the genericity condition; the procedure is repeated until the genericity condition is satisfied. Note that the set $Z:=V\left(G_{1}, \ldots, G_{r}\right)$ has pure codimension $r$ and $X \subset Z$. Let $\gamma_{1}, \ldots, \gamma_{\tau}$ be some (random) generic rational numbers and set

$$
H:= \begin{cases}1 & \text { if } W=\emptyset \\ \sum_{i=1}^{\tau} \gamma_{i} u_{i} & \text { otherwise }\end{cases}
$$

Clearly $\operatorname{dim}(X \cap V(H))<\operatorname{dim} X$. Moreover, for a sufficiently general linear $r$ dimensional subspace $L^{r} \subset \mathbb{C}^{n}$, the intersection $L^{r} \cap Z$ has only isolated smooth points and $L^{r} \cap Y_{i} \neq \emptyset$ for every $i=1, \ldots, m$. We can assume that $L^{r}$ is determined by the linear forms $l_{i}=\sum_{j=1}^{n} \beta_{i j} x_{j}, i=1, \ldots, n-r$, where $\beta_{i j}$ are sufficiently 
generic (random) rational numbers. Now take

$$
p_{X, W}=\left|\operatorname{Jac}\left(G_{1}, \ldots, G_{r}, l_{1}, \ldots, l_{n-r}\right)\right| \cdot H,
$$

where $\mathrm{Jac}(\cdot)$ denotes the Jacobian matrix. Then $p_{X, W}$ is a polynomial with the required properties.

Remark 2.2 Theoretically, a random rational number is a generic rational number, but practically by random numbers we mean rational numbers produced by special random algorithms.

The polynomial $p_{X, W}$ can be found by using a probabilistic algorithm. First recall the following:

Definition 2.3 Let $I$ be an ideal in $\mathbb{C}\left[x_{1}, \ldots, x_{n}\right]$. We define the homogenization of $I$ to be the ideal $I^{\mathrm{h}}$ generated by $\left\{f^{\mathrm{h}}: f \in I\right\} \subset \mathbb{C}\left[x_{0}, \ldots, x_{n}\right]$, where $f^{\mathrm{h}}$ is the homogenization of $f$.

Theorem 2.4 ([2, Thm. 4, §4, Chap. 8, p. 388]) Let I be an ideal in $k\left[x_{1}, \ldots, x_{n}\right]$ and let $G=\left\{g_{1}, \ldots, g_{s}\right\}$ be a Gröbner basis for $I$ with respect to a graded lexicographic order in $k\left[x_{1}, \ldots, x_{n}\right]$ (i.e., the lexicographic order that first compares the total degree: $x^{\alpha}>x^{\beta}$ whenever $\left.|\alpha|>|\beta|\right)$. Then $G^{\mathrm{h}}=\left\{g_{1}^{\mathrm{h}}, \ldots, g_{s}^{\mathrm{h}}\right\}$ is a basis for $I^{\mathrm{h}} \subset k\left[x_{0}, x_{1}, \ldots, x_{n}\right]$.

This theorem allows us to compute the set of points at infinity of an affine variety given by the ideal $I$; to this aim, it is enough to compute the Gröbner basis $\left\{g_{1}, \ldots, g_{s}\right\}$ of the ideal $I$ and then to consider the ideal $I_{\infty}=\left(x_{0}, g_{1}^{\mathrm{h}}, \ldots, g_{s}^{\mathrm{h}}\right)$. In particular, we can check effectively whether $L^{r} \cap \bar{X} \cap\left\{x_{0}=0\right\}=\emptyset$, which implies that $L^{r} \cap Y_{i} \neq \emptyset$ for $i=1, \ldots, m$ (see the proof of Proposition 2.1). This is crucial for our computations.

Now we sketch the algorithm to compute the polynomial $p_{X, W}$. Note that, for a given ideal $I$, we can compute $\operatorname{dim} V(I)$ by [19].

INPUT: The ideal $I=I(X)=\left(g_{1}, \ldots, g_{\omega}\right)$ and the ideal $J=I(W)=$ $\left(g_{1}, \ldots, g_{\omega}, u_{1}, \ldots, u_{\tau}\right)$

1) repeat

choose random rational numbers $\alpha_{i 1}, \ldots, \alpha_{i \omega}, i=1, \ldots, r$;

put $G_{i}:=\sum_{k=1}^{\omega} \alpha_{i k} g_{k}, i=1, \ldots, r$;

put $I=\left(G_{1}, \ldots, G_{r}\right)$;

until $\operatorname{dim} V(I)=n-r$.

2) repeat

choose random rational numbers $\beta_{i 1}, \ldots, \beta_{i n}, i=1, \ldots, n-r$;

put $l_{i}:=\sum_{k=1}^{n} \beta_{i k} x_{k}, i=1, \ldots, n-r$;

put $I=\left(G_{1}, \ldots, G_{r}, l_{1}, \ldots, l_{n-r}\right)$;

compute the ideal $I_{\infty}=\left(H_{1}, \ldots, H_{m}\right) \subset k\left[x_{0}, \ldots, x_{n}\right]$;

if $\operatorname{dim} V\left(I_{\infty}\right)=0$ then

begin

compute $V\left(G_{1}, \ldots, G_{r}, l_{1}, \ldots, l_{r}\right):=\left\{a_{1}, \ldots, a_{p}\right\}$

end 
until $\operatorname{dim} V\left(I_{\infty}\right)=0$ and $\left|\operatorname{Jac}\left(G_{1}, \ldots, G_{r}, l_{1}, \ldots, l_{n-r}\right)\left(a_{i}\right)\right| \neq 0$ for $i=$ $1, \ldots p$.

3) repeat

choose random rational numbers $\gamma_{1}, \ldots, \gamma_{\tau}$;

put $H:=\sum_{k=1}^{\tau} \gamma_{i} u_{k}$;

put $J=\left(G_{1}, \ldots, G_{r}, H\right)$;

until $\operatorname{dim} V(J)<n-r$.

OUTPUT: $p_{X, W}=\left|\operatorname{Jac}\left(G_{1}, \ldots, G_{r}, l_{1}, \ldots, l_{n-r}\right)\right| \cdot H$

Remark 2.5 Let us assume that $I(X)$ and $I(W)$ are generated by polynomials from the ring $\mathbb{F}\left[x_{1}, \ldots, x_{n}\right]$, where $\mathbb{F}$ is a subfield of $\mathbb{C}$. Then we can choose a polynomial $p_{X, W}$ such that $p_{X, W} \in \mathbb{F}\left[x_{1}, \ldots, x_{n}\right]$.

From the proof of Proposition 2.1, with no loss of generality, we can assume that $\operatorname{rank} \operatorname{Jac}\left(g_{1}, \ldots, g_{r}\right)=r$ on some nonempty regular open subset $X^{0}$ of $X$ and that $X=\overline{X^{0}}$. It is clear that $V\left(p_{X, W}\right)$ contains $\operatorname{sing}(X) \cup W$ and the singular points of the projection $\left(l_{1}, \ldots, l_{n-r}\right): X \rightarrow \mathbb{C}^{n-r}$. Now, to construct an affine stratification of $X$, it is enough to construct an affine filtration $X=X_{0} \supset X_{1} \supset \cdots \supset X_{n-r} \supset$ $X_{n-r+1}=\emptyset$ by induction with $X_{i+1}:=X_{i} \cap V\left(p_{X_{i}, \emptyset}\right), i=0, \ldots, n-r$. The degree of each $X_{i}$ can be calculated and depends only on $D$.

\subsection{Construction of Affine Whitney Stratifications}

In this section, we construct an affine Whitney stratification of a given affine variety $X$, with $I(X)=\left(g_{1}, \ldots, g_{r}\right)$ and $\operatorname{deg} g_{i} \leq D$, by refining the affine stratification given in Sect. 2.2 so that the resulting stratification is still affine and moreover satisfies the Whitney condition.

First of all, inspired by the construction in [5,17], let us describe the Whitney condition (b) algebraically. Assume that $Y \subset X$ is an affine subvariety of $X$ with $\operatorname{dim} Y<\operatorname{dim} X$ defined by

$$
Y:=X \cap\left\{\widetilde{g}_{r+1}=\cdots=\widetilde{g}_{p}=0\right\} .
$$

Set

$$
\Gamma_{1}:=\left\{\begin{array}{l}
(x, y, w, v, \gamma, \lambda) \in \mathbb{C}^{n} \times \mathbb{C}^{n} \times \mathbb{C}^{n} \times \mathbb{C}^{n} \times \mathbb{C} \times \mathbb{C}^{r}: \\
g_{1}(x)=\cdots=g_{r}(x)=0 \\
g_{1}(y)=\cdots=g_{r}(y)=\widetilde{g}_{r+1}(y)=\cdots=\widetilde{g}_{p}(y)=0 \\
w=\gamma(x-y) \\
v=\sum_{i=1}^{r} \lambda_{i} \mathrm{~d}_{x} g_{i}
\end{array}\right\},
$$

and let

$$
\pi_{1}: \mathbb{C}^{n} \times \mathbb{C}^{n} \times \mathbb{C}^{n} \times \mathbb{C}^{n} \times \mathbb{C} \times \mathbb{C}^{r} \rightarrow \mathbb{C}^{n} \times \mathbb{C}^{n} \times \mathbb{C}^{n} \times \mathbb{C}^{n}
$$

be the projection on the first $4 n$ coordinates. Let $C(X, Y)=\overline{\pi_{1}\left(\Gamma_{1}\right)} \mathcal{Z} \subset(X \times Y \times$ $\mathbb{C}^{n} \times \mathbb{C}^{n}$ ), where the closure is taken in the Zariski topology (which coincides with the 
topological closure; see, e.g., [16, Prop. 7]). Of course, $C(X, Y)$ is an affine variety. We have the following:

Lemma 2.6 For each $(x, x, w, v) \in C(X, Y)$, there are sequences $x^{k} \in X^{0}, y^{k} \in Y$, $\gamma^{k} \in \mathbb{C}$, and $\lambda^{k} \in \mathbb{C}^{r}$ such that:

- $x^{k} \rightarrow x$,

- $y^{k} \rightarrow x$,

- $w^{k}:=\gamma^{k}\left(x^{k}-y^{k}\right) \rightarrow w$,

- $v^{k}:=\sum_{i=1}^{r} \lambda_{i}^{k} \mathrm{~d}_{x^{k}} g_{i} \rightarrow v$.

Proof By construction, there are sequences $\bar{x}^{k} \in X, y^{k} \in Y, \bar{\gamma}^{k} \in \mathbb{C}$, and $\lambda^{k} \in \mathbb{C}^{r}$ such that $\bar{x}^{k}, y^{k} \rightarrow x, \bar{w}^{k}:=\bar{\gamma}^{k}\left(\bar{x}^{k}-y^{k}\right) \rightarrow w$, and $\sum_{i=1}^{r} \lambda_{i}^{k} \mathrm{~d}_{\bar{x}^{k}} g_{i} \rightarrow v$. It is clear that, by taking subsequences if necessary, we may suppose that:

- either $\bar{x}^{k}=y^{k}$ for every $k$ or $\bar{x}^{k} \neq y^{k}$ for every $k$,

- for each $i$, either $\lambda_{i}^{k} \neq 0$ for every $k$ or $\lambda_{i}^{k}=0$ for every $k$.

Set

$$
\gamma^{k}= \begin{cases}0 & \text { if } \bar{x}^{k}=y^{k} \text { for every } k \\ \bar{\gamma}^{k} & \text { if } \bar{x}^{k} \neq y^{k} \text { for every } k\end{cases}
$$

Suppose that $\lambda_{i}^{k} \neq 0$ for $i=1, \ldots, r^{\prime} \leqslant r, k \in \mathbb{N}$ and $\lambda_{i}^{k}=0$ for $i=r^{\prime}+$ $1, \ldots, r, k \in \mathbb{N}$. Since $\bar{x}^{k} \in \overline{X^{0}}$, there exists a sequence $x^{k} \in X^{0}$ such that

$$
\left\|x^{k}-\bar{x}^{k}\right\| \leqslant \begin{cases}\frac{1}{k} & \text { if } \bar{x}^{k}=y^{k} \text { for every } k \\ \frac{\left\|\bar{x}^{k}-y^{k}\right\|}{k} & \text { if } \bar{x}^{k} \neq y^{k} \text { for every } k\end{cases}
$$

so $x^{k} \rightarrow x$. By continuity, we can also choose $x^{k}$ so that $\left\|\mathrm{d}_{x^{k}} g_{i}-\mathrm{d}_{\bar{x}^{k}} g_{i}\right\|<\frac{1}{k \lambda_{i}^{k}}$ if $\lambda_{i}^{k} \neq 0$. Set $v^{k}:=\sum_{i=1}^{r} \lambda_{i}^{k} \mathrm{~d}_{x^{k}} g_{i}$. Then

$$
\begin{aligned}
\left\|v^{k}-\sum_{i=1}^{r} \lambda_{i}^{k} \mathrm{~d}_{\bar{x}^{k}} g_{i}\right\| & =\left\|\sum_{i=1}^{r^{\prime}} \lambda_{i}^{k}\left(\mathrm{~d}_{x^{k}} g_{i}-\mathrm{d}_{\bar{x}^{k}} g_{i}\right)\right\| \\
& \leqslant \sum_{i=1}^{r^{\prime}}\left|\lambda_{i}^{k}\right|\left\|\mathrm{d}_{x^{k}} g_{i}-\mathrm{d}_{\bar{x}^{k}} g_{i}\right\|<\frac{r^{\prime}}{k} \rightarrow 0,
\end{aligned}
$$

i.e., $v^{k} \rightarrow v$. Set $w^{k}:=\gamma^{k}\left(x^{k}-y^{k}\right)$. Now, if $\bar{x}^{k}=y^{k}$ for every $k$, then $\gamma^{k}=0$ and $w=\bar{w}^{k}=0$, so we have $w^{k}=0=w$. If $\bar{x}^{k} \neq y^{k}$ for every $k$, then

$$
\left\|w^{k}-\bar{w}^{k}\right\|=\left|\gamma^{k}\right| \cdot\left\|\left(x^{k}-\bar{x}^{k}\right)\right\| \leqslant\left|\gamma^{k}\right| \cdot \frac{\left\|\bar{x}^{k}-y^{k}\right\|}{k}=\frac{\left\|\bar{w}^{k}\right\|}{k} \rightarrow 0 .
$$

Hence $w^{k} \rightarrow w$ 
The following algebraic criterion permits us to check Whitney regularity on $Y^{0}=$ $Y \backslash V\left(p_{Y, W}\right)$, where the notation $V\left(p_{Y, W}\right)$ is from Proposition 2.1 and the affine set $W$ will be determined later.

Lemma 2.7 Let $x \in Y^{0}$. Then the pair $\left(X^{0}, Y^{0}\right)$ satisfies the Whitney condition (b) at $x$ if and only if, for any $(x, x, w, v) \in C(X, Y)$, we have $v \cdot w=0$.

Proof Suppose that $\left(X^{0}, Y^{0}\right)$ is Whitney regular at $x$ and assume for contradiction that there is $(x, x, w, v) \in C(X, Y)$ such that $v \cdot w \neq 0$. In view of Lemma 2.6, there are sequences $x^{k} \in X^{0}, y^{k} \in Y, \gamma^{k} \in \mathbb{C}$, and $\lambda^{k} \in \mathbb{C}^{r}$ such that

- $x^{k} \rightarrow x, y^{k} \rightarrow x$

- $w^{k}:=\gamma^{k}\left(x^{k}-y^{k}\right) \rightarrow w$,

- $v^{k}:=\sum_{i=1}^{r} \lambda_{i}^{k} \mathrm{~d}_{x^{k}} g_{i} \rightarrow v$.

Note that $w \neq 0$, so $w$ determines the limit of the sequence of secants $\overline{x^{k} y^{k}}$ and it follows that $x^{k} \neq y^{k}$ for $k$ large enough. By taking a subsequence if necessary, we may assume that $T_{x^{k}} X^{0} \rightarrow T$. By assumption, $w \in T$. For each $k$, let $\left\{b_{1}^{k}, \ldots, b_{r}^{k}\right\}$ be an orthonormal basis of $N_{x^{k}} X^{0}$; recall that $N_{x^{k}} X^{0}:=\operatorname{span}\left\{\overline{\mathrm{d}_{x^{k}} g_{1}}, \ldots, \overline{\mathrm{d}_{x^{k}} g_{r}}\right\}$ is the normal space of $X^{0}$ at $x^{k}$. Obviously $\left\langle\overline{b_{1}^{k}}, \ldots, \overline{b_{r}^{k}}\right\rangle^{\perp}=T_{x^{k}} X^{0}$. By compactness, each sequence $b_{i}^{k}$ has an accumulation point $b_{i}$. Without loss of generality, suppose that $b_{i}^{k} \rightarrow b_{i}$. It is clear that the system $\left\{b_{1}, \ldots, b_{r}\right\}$ is also orthonormal and $\left\langle\bar{b}_{1}, \ldots, \bar{b}_{r}\right\rangle^{\perp}=T$. Let $\tilde{\lambda}^{k}=\left(\tilde{\lambda}_{1}^{k}, \ldots, \widetilde{\lambda}_{r}^{k}\right)$ be such that $v^{k}:=\sum_{i=1}^{r} \tilde{\lambda}_{i}^{k} b_{i}^{k}$. Then $\tilde{\lambda}^{k}$ is convergent to a limit $\tilde{\lambda}$ and it is clear that $v=\sum_{i=1}^{r} \tilde{\lambda}_{i} b_{i}$. Finally, we have $w \in T=\left\langle\overline{b_{1}}, \ldots, \overline{b_{r}}\right\rangle^{\perp} \subset\langle\bar{v}\rangle^{\perp}$, i.e., $v \cdot w=0$, which is a contradiction.

Now suppose that $v \cdot w=0$ for any $(x, x, w, v) \in C(X, Y)$ and assume that $\left(X^{0}, Y^{0}\right)$ is not Whitney regular at $x$. So, there are sequences $x^{k} \in X^{0}$ and $y^{k} \in Y^{0}$ with the following properties:

- $x^{k} \neq y^{k}, x^{k} \rightarrow x, y^{k} \rightarrow y$;

- $T_{x^{k}} X^{0} \rightarrow T$;

- the sequence of secants $\overline{x^{k} y^{k}}$ tends to a line $\ell \not \subset T$.

For each $k$, let $\left\{b_{1}^{k}, \ldots, b_{r}^{k}\right\}$ be an orthonormal basis of $N_{x^{k}} X^{0}$ so $\left\langle\overline{b_{1}^{k}}, \ldots, \overline{b_{r}^{k}}\right\rangle^{\perp}=$ $T_{x^{k}} X^{0}$. As above, we may assume that $b_{i}^{k} \rightarrow b_{i}$. Then the system $\left\{b_{1}, \ldots, b_{r}\right\}$ is also orthonormal and $\left\langle\overline{b_{1}}, \ldots, \overline{b_{r}}\right\rangle^{\perp}=T$. Let $w^{k}:=\frac{x^{k}-y^{k}}{\left\|x^{k}-y^{k}\right\|}$; we can assume that the limit $w:=\lim w^{k}$ exists, and clearly $w$ is a direction vector of $\ell$. By assumption, $w \notin T=\left\langle\bar{b}_{1}, \ldots, \bar{b}_{r}\right\rangle^{\perp}$; i.e., there exists an index $j$ such that $b_{j} \cdot w \neq 0$. To obtain a contradiction, it is enough to show that there is a sequence $v^{k}:=\sum_{i=1}^{r} \lambda_{i}^{k} \mathrm{~d}_{x^{k}} g_{i}$ such that $v^{k} \rightarrow b_{j}$, but this is clear since $b_{j}^{k} \in \operatorname{span}\left\{\mathrm{d}_{x^{k}} g_{1}, \ldots, \mathrm{d}_{x^{k}} g_{r}\right\}$, so such a sequence always exists.

Now, according to [11, Algorithm 3.3], [7, Algorithm 4.5.3] (see also [4,6]), it is possible to calculate a basis for the ideal $I\left(\Gamma_{1}\right)$ by calculating the radical of the 
following ideal in $\mathbb{C}[x, y, w, v, \gamma, \lambda]$ :

$$
\left(\begin{array}{l}
g_{1}(x), \ldots, g_{r}(x) \\
g_{1}(y), \ldots, g_{r}(y), \widetilde{g}_{r+1}(y), \ldots, \widetilde{g}_{p}(y), \\
w-\gamma(x-y) \\
v-\sum_{i=1}^{r} \lambda_{i} \mathrm{~d}_{x} g_{i}
\end{array}\right) .
$$

Then, by Buchberger's algorithm, we can calculate a Gröbner basis of $I\left(\Gamma_{1}\right)$. So, in view of [10, Thm. 5.1], [14], we can compute a Gröbner basis of the ideal $I(C(X, Y))$. Now we give another criterion for Whitney regularity.

Lemma $2.8 \operatorname{Let}\left\{h_{1}(x, y, w, v), \ldots, h_{q}(x, y, w, v)\right\}$ be a Gröbner basis of $I(C(X, Y))$ and set

$$
\Gamma_{2}:=\left\{\begin{array}{l}
(x, x, w, v, \gamma, \lambda) \in \mathbb{C}^{n} \times \mathbb{C}^{n} \times \mathbb{C}^{n} \times \mathbb{C}^{n} \times \mathbb{C} \times \mathbb{C}: \\
h_{1}(x, x, w, v)=\cdots=h_{q}(x, x, w, v)=0 \\
\gamma \sum_{j=1}^{n} v_{j} w_{j}=1 \\
\lambda p_{Y, \emptyset}(x)=1
\end{array}\right\},
$$

where $p_{Y, \emptyset}(x)$ is the polynomial determined in Proposition 2.1. Let $Y^{0}:=Y \backslash V\left(p_{Y, \emptyset}\right)$. Then the pair $\left(X^{0}, Y^{0}\right)$ is not Whitney regular at $x$ if and only if there exists $(w, v, \gamma, \lambda) \in \mathbb{C}^{n} \times \mathbb{C}^{n} \times \mathbb{C} \times \mathbb{C}$ such that $(x, x, w, v, \gamma, \lambda) \in \Gamma_{2}$.

Proof Note that $x \in Y^{0}$ if and only if $p_{Y, \emptyset}(x) \neq 0$, i.e., there exists $\lambda \in \mathbb{C}$ such that $\lambda p_{Y, \emptyset}(x)=1$. In view of Lemma 2.7 , the pair $\left(X^{0}, Y^{0}\right)$ is not Whitney regular at $x$ if and only if there exist $w, v$ with $v \cdot w \neq 0$ such that $(x, x, w, v) \in C(X, Y)$. The lemma follows easily.

Now we determine an algebraic set $W=W(X, Y)$ in $Y$ with $\operatorname{dim} W<\operatorname{dim} Y$ and $V\left(p_{Y, \emptyset}\right) \subset W$ such that the pair $\left(X^{0}, Y \backslash W\right)$ is Whitney regular. Let

$$
\pi_{2}: \mathbb{C}^{n} \times \mathbb{C}^{n} \times \mathbb{C}^{n} \times \mathbb{C}^{n} \times \mathbb{C} \times \mathbb{C} \rightarrow \mathbb{C}^{n}
$$

be the projection on the first $n$ coordinates. By Lemma 2.8, $\pi_{2}\left(\Gamma_{2}\right)$ is the set of points where the Whitney condition (b) fails to be satisfied. Let $\frac{\pi_{2}\left(\Gamma_{2}\right)}{\mathcal{Z}}$ be the Zariski closure of $\pi_{2}\left(\Gamma_{2}\right)$, then $\overline{\pi_{2}\left(\Gamma_{2}\right)} \mathcal{Z}$ is affine. It follows from [21, Thm. 8.5], [22, Lem. 19.3] that $\operatorname{dim} \pi_{2}\left(\Gamma_{2}\right)<\operatorname{dim} Y$, so $\operatorname{dim} \overline{\pi_{2}\left(\Gamma_{2}\right)} \mathcal{Z}<\operatorname{dim} Y$. Set

$$
W=W(X, Y):=\overline{\pi_{2}\left(\Gamma_{2}\right)} \mathcal{Z}
$$

then obviously $\operatorname{dim} W<\operatorname{dim} Y$. Again, applying [11, Algorithm 3.3] or [7, Algorithm 4.5.3] (see also $[4,6]$ ) to find a system of generators of $I(W)$, then applying [10, Thm. 5.1], [14], we can compute a Gröbner basis of the ideal $I(W)$. Finally, let

- $X_{0}:=X$,

- $X_{1}:=X_{0} \cap V\left(p_{X_{0}, \emptyset}\right)$,

- $X_{2}:=X_{1} \cap V\left(p_{X_{1}, W\left(X_{0}, X_{1}\right)}\right)$, 
- $X_{3}:=X_{2} \cap V\left(p_{X_{2}, W\left(X_{0}, X_{2}\right) \cup W\left(X_{1}, X_{2}\right)}\right), \ldots$,

- $X_{i}:=X_{i-1} \cap V\left(p_{X_{i-1}, \bigcup_{j=0}^{i-2} W\left(X_{j}, X_{i-1}\right)}\right), \ldots$

By induction, we can construct a finite filtration of algebraic sets $X=X_{0} \supset X_{1}$ $\supset \cdots \supset X_{n-r} \supset X_{n-r+1}=\emptyset$ with $\operatorname{dim} X_{i}>\operatorname{dim} X_{i+1}$. Let $B_{i}:=X_{i} \backslash X_{i+1}$. Then $\mathcal{S}:=\left\{B_{i}\right\}_{i=1, \ldots, q}$ is a Whitney stratification of $X$. Note that the degree of $X_{i}$ can be determined explicitly and depends only on $D$.

\section{Thom Isotopy Lemma for Nonproper Maps}

We start this section with:

Definition 3.1 Let $f: X \rightarrow \mathbb{C}^{m}$ be a polynomial dominant map, where $X$ is an algebraic set. Let $\mathcal{S}=\left\{X_{\alpha}\right\}_{\alpha \in I}$ be a stratification of $X$. By $K_{\infty}\left(f, X_{\alpha}\right)$ we mean the set $\left\{y \in \mathbb{C}^{m}:\right.$ there is a sequence $x^{k} \rightarrow \infty, x^{k} \in X_{\alpha}$ such that $\left\|x^{k}\right\| v\left(\mathrm{~d}_{x^{k}}\left(\left.f\right|_{X_{\alpha}}\right)\right) \rightarrow$ 0 and $\left.f\left(x^{k}\right) \rightarrow y\right\}$ (here $v$ denotes the Rabier function; for details, see [10, Sect. 5]). Now, let $C\left(f, X_{\alpha}\right)$ denote the set of points where $\left.f\right|_{X_{\alpha}}$ is not a submersion. By $\operatorname{sing}(f, \mathcal{S})$ we denote the set of stratified singular values of $f$, i.e.,

$$
\operatorname{sing}(f, \mathcal{S})=\bigcup_{\alpha \in I} K_{0}\left(f, X_{\alpha}\right)
$$

where $K_{0}\left(f, X_{\alpha}\right)=\overline{f\left(C\left(f, X_{\alpha}\right)\right)}$.

By [10, Thm. 3.3], we have that, for every $\alpha$, the set $K_{\infty}\left(\left.f\right|_{X_{\alpha}}\right)$ is closed and has measure 0 in $\mathbb{C}^{m}$. In particular the set $K(f)$ defined below is also closed and has measure 0 .

Definition 3.2 Let $K(f, \mathcal{S})$ be the set of stratified generalized critical values of $f$ given by

$$
K(f, \mathcal{S}):=\bigcup_{\alpha \in I}\left(K_{0}\left(f, X_{\alpha}\right) \cup K_{\infty}\left(f, X_{\alpha}\right)\right) .
$$

Remark 3.3 The set $K(f, \mathcal{S})$ is closed. Indeed, it is enough to see that $K_{\infty}\left(f, X_{\alpha}\right)$ is closed for every $\alpha$. Assume that $y^{k} \in K_{\infty}\left(f, X_{\alpha}\right)$ and $y^{k} \rightarrow y$. So, for every $k$, there are suitable sequences $x^{k j} \in X_{\alpha}, j=1,2, \ldots$ such that $\lim _{j \rightarrow \infty} f\left(x^{k j}\right)=y^{k}$. Hence, for any $k$, we can choose $x^{k j_{k}}$ such that:

- $\left\|f\left(x^{k j_{k}}\right)-y^{k}\right\|<1 / k$,

- $\left\|x^{k j_{k}}\right\|>k$,

- $\left\|x^{k j_{k}}\right\| v\left(\mathrm{~d}_{x^{k j_{k}}} f\right)<1 / k$.

Set $z^{k}:=x^{k j_{k}}$. Thus $z^{k} \in X_{\alpha}, z^{k} \rightarrow \infty,\left\|z^{k}\right\| v\left(\mathrm{~d}_{z^{k}}\left(\left.f\right|_{X_{\alpha}}\right)\right) \rightarrow 0$, and $f\left(z^{k}\right) \rightarrow y$. Consequently $y \in K_{\infty}\left(f, X_{\alpha}\right)$.

Assuming that $\mathcal{S}$ is an affine Whitney stratification of $X$, we prove that $K(f, \mathcal{S})$ contains the set of bifurcation values of $f$. 
Theorem 3.4 (First isotopy lemma for nonproper maps) Let $X \subset \mathbb{C}^{n}$ be an affine variety with an affine Whitney stratification $\mathcal{S}$, and let $f=\left(f_{1}, \ldots, f_{m}\right): X \rightarrow \mathbb{C}^{m}$ be a polynomial dominant map. Let $K(f, \mathcal{S})$ be the set of stratified generalized critical values of $f$ given by (2). Then $f$ is locally trivial outside $K(f, \mathcal{S})$.

Before proving Theorem 3.4, recall that the Whitney condition (b) is equivalent to the condition (w) (see [17, V.1.2]), so it is more convenient to use the condition (w), since we will need to construct rugose vector fields in the sense of [20]. In what follows, it is more convenient to work with the underlying real algebraic set of $X$ in $\mathbb{R}^{2 n}$, also denoted by $X$; the affine Whitney stratification $\mathcal{S}$ of $X$ induces a semialgebraic Whitney stratification of the underlying set with the corresponding strata denoted by the same notation $X_{\beta}$. We also identify the polynomial map $f$ with the real polynomial map $\left(\operatorname{Re} f_{1}, \ldots, \operatorname{Re} f_{m}, \operatorname{Im} f_{1}, \ldots, \operatorname{Im} f_{m}\right): X \rightarrow \mathbb{R}^{2 m}$. Let us recall the definitions pertaining to rugosity. Let $\psi: X \rightarrow \mathbb{R}$ be a real function. We say that $\psi$ is a rugose function if the following conditions are fulfilled:

- The restriction $\left.\psi\right|_{X_{\beta}}$ to any stratum $X_{\beta}$ is a smooth function.

- For any stratum $X_{\beta}$ and for any $x \in X_{\beta}$, there exist a neighborhood $U$ of $x$ in $\mathbb{R}^{2 n}$ and a constant $c>0$ such that, for any $y \in X \cap U$ and $x^{\prime} \in X_{\beta} \cap U$, we have $\left|\psi(y)-\psi\left(x^{\prime}\right)\right| \leqslant c\left\|y-x^{\prime}\right\|$.

A rugose map is a map whose components are rugose functions. A vector field $v$ on $X$ is called a rugose vector field if $v$ is a rugose map and $v(x)$ is tangent to the stratum containing $x$ for any $x \in X$.

Proof of Theorem 3.4 Let $z \in \mathbb{C}^{m} \backslash K(f, \mathcal{S})$, where we identify $\mathbb{C}^{m}$ with $\mathbb{R}^{2 m}$, and let $B$ be an open box centered at $z$ such that $\bar{B} \cap K(f, \mathcal{S})=\emptyset$. To prove the theorem, it is enough to prove that $f$ is trivial on $B$. Without loss of generality, we may suppose that $z=0$ and $B=(-1,1)^{2 m}$. Let $\partial_{1}, \ldots, \partial_{2 m}$ be the restrictions of the coordinate vector fields (on $\mathbb{R}^{2 m}$ ) to $\bar{B}$. Set $U:=f^{-1}(B), U_{\beta}:=U \cap X_{\beta}$, and

$$
I^{\prime}:=\left\{\beta \in I: U_{\beta} \neq \emptyset\right\} .
$$

Clearly $\bar{U}=f^{-1}(\bar{B})$ and $I^{\prime}=\left\{\beta \in I: \bar{U} \cap X_{\beta} \neq \emptyset\right\}$. First of all, let us give a sufficient condition for trivializing a rugose vector field.

Lemma 3.5 For $i=1, \ldots, 2 m$, let $v_{i}$ be vector fields on $X$ which are rugose in $U$. Assume that $\mathrm{d} f\left(v_{i}\right)=\partial_{i}$ and there is a positive constant $c>0$ such that $\left\|v_{i}(x)\right\| \leqslant$ $\frac{\|x\|+1}{c}$ for any $x \in U$. Then $f$ is a topologically trivial fibration over $B$.

Proof It is enough to prove that there is a homeomorphism $\phi: f^{-1}(B) \rightarrow f^{-1}(0) \times B$ such that the following diagram commutes:

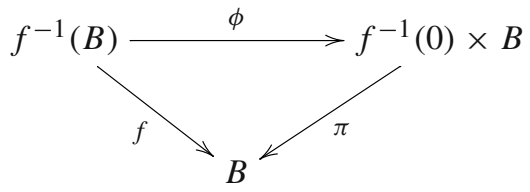

where $\pi$ denotes the projection on the second factor. We note the following facts: 
(i) The flow of $v_{i}$ preserves the stratification. This is a consequence of the rugosity. For more detail, see [20, Prop. 4.8].

(ii) For each $i$ and any $x \in U$, there is a unique integral curve of $v_{i}$ passing through $x$. This follows from the uniqueness of integral curves of smooth vector fields and the fact that $v_{i}$ preserves the stratification.

Set $Y_{t}^{i}:=\left(y_{1}, \ldots, y_{i-1}, t, y_{i+1}, \ldots, y_{n}\right)$ and $Y^{i}=\left\{Y_{t}^{i}: t \in(-1,1)\right\}$. First of all, we will prove that the flow of $v_{i}$ induces a homeomorphism $\phi_{i}: f^{-1}\left(Y^{i}\right) \rightarrow$ $f^{-1}\left(Y_{0}^{i}\right) \times(-1,1)$ such that the following diagram commutes:

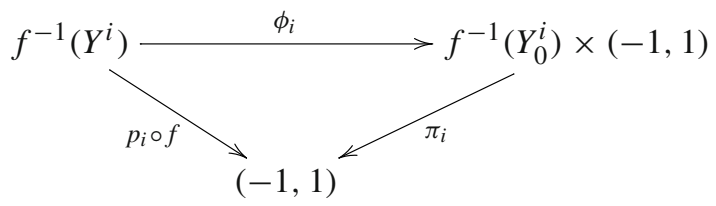

where $\pi_{i}$ denotes the projection on the second factor and $p_{i}$ denotes the projection on the $i^{\text {th }}$ coordinate. This follows from the following claim which states that there is no trajectory of $v_{i}$ going to infinity:

Claim 3.6 For each $x \in f^{-1}\left(Y_{0}^{i}\right)$, let $\gamma$ be the integral curve of $v_{i}$ such that $\gamma(0)=x$. Then $\gamma$ reaches any level $f^{-1}\left(Y_{t}^{i}\right)$ at time $t$ for $t \in(-1,1)$.

Proof By assumption, $\|\dot{\gamma}(t)\| \leqslant \frac{\|\gamma(t)\|+1}{c}$. Without loss of generality, suppose that $t>0$. In light of the Gronwall lemma, by repeating the calculation of [1, Thm. 3.5], we obtain

$$
\begin{aligned}
\|\gamma(t)\| & \leqslant\|\gamma(0)\|+\int_{0}^{t} \frac{\|\gamma(s)\|+1}{c} \mathrm{~d} s \\
& =\|x\|+\frac{t}{c}+\int_{0}^{t} \frac{\|\gamma(s)\|}{c} \mathrm{~d} s \\
& \leqslant\left(\|x\|+\frac{t}{c}\right) \exp \int_{0}^{t} \frac{\mathrm{d} s}{c}=\left(\|x\|+\frac{t}{c}\right) \mathrm{e}^{t / c}<+\infty,
\end{aligned}
$$

which implies that the trajectory $\gamma$ does not go to infinity at time $t$. Now we have

$$
\begin{aligned}
f(\gamma(t))-f(x) & =\int_{0}^{t} \mathrm{~d}[f(\gamma(t))] \\
& =\int_{0}^{t} \mathrm{~d}_{\gamma(t)} f(\dot{\gamma}(t)) \mathrm{d} t=\int_{0}^{t} \partial_{i} \mathrm{~d} t=(0, \ldots 0, t, 0, \ldots, 0) .
\end{aligned}
$$

Since $f(x) \in Y_{0}^{i}$, clearly $f(\gamma(t)) \in Y_{t}^{i}$, and the claim follows.

For any $x \in f^{-1}\left(Y_{0}^{i}\right)$, let $h_{i}(x, t)=x+\int_{0}^{t} \dot{\gamma}(s) \mathrm{d} s$. Then $h_{i}$ defines a homeomorphism $f^{-1}\left(Y_{0}^{i}\right) \times(-1,1) \rightarrow f^{-1}\left(Y^{i}\right)$. Then $\phi_{i}=h_{i}^{-1}$ is the required homeomorphism. Now, for $x \in f^{-1}(0)$, let $h: f^{-1}(0) \times B \rightarrow f^{-1}(B)$ be defined by

$$
h\left(x, t_{1}, \ldots, t_{2 m}\right)=h_{2 m}\left(\ldots\left(h_{2}\left(h_{1}\left(x, t_{1}\right), t_{2}\right), \ldots, t_{2 m}\right) .\right.
$$


Then $\phi:=h^{-1}$ is a homeomorphism, as required. The lemma is proved.

Now let us prove the following:

Lemma 3.7 There is a constant $c>0$ such that, for any $\beta \in I^{\prime}$ and any $x \in \bar{U} \cap X_{\beta}$, we have

$$
(\|x\|+1) v\left(\mathrm{~d}_{x}\left(\left.f\right|_{X_{\beta}}\right)\right) \geqslant c .
$$

Proof Assume for contradiction that there exist an index $\beta \in I^{\prime}$ and a sequence $x^{k} \in \bar{U} \cap X_{\beta}$ such that $\left(\left\|x^{k}\right\|+1\right) v\left(\mathrm{~d}_{x^{k}}\left(\left.f\right|_{X_{\beta}}\right)\right) \rightarrow 0$. Taking a subsequence if necessary, we can suppose that $x^{k} \rightarrow x, T_{x^{k}} X_{\beta} \rightarrow T$ and $f\left(x^{k}\right) \rightarrow y \in \bar{B}$ with $x \in \mathbb{C}^{n}$ or $x=\infty$. If $x=\infty$, then by definition, $y \in K_{\infty}\left(\left.f\right|_{X_{\beta}}\right) \subset K(f, \mathcal{S})$. This is a contradiction since $\bar{B} \cap K(f, \mathcal{S})=\emptyset$. Thus $x \in \mathbb{C}^{n}$, and we get $v\left(\mathrm{~d}_{x^{k}}\left(\left.f\right|_{X_{\beta}}\right)\right) \rightarrow 0$. In the case $x \in X_{\beta}$, in view of [15, Lem. 2.2], we have $v\left(\mathrm{~d}_{x}\left(\left.f\right|_{X_{\beta}}\right)\right)=0$, i.e., $y \in K_{0}\left(f, X_{\beta}\right)$, which is also a contradiction. Therefore $x \in \bar{X}_{\beta} \backslash X_{\beta}$. Denote by $X_{\alpha}$ the stratum containing $x$. Let $F=\left(f_{1}, \ldots, f_{m}\right): \mathbb{C}^{n} \rightarrow \mathbb{C}^{m}$ be the polynomial extending $f$ on $\mathbb{C}^{n}$. Obviously $v\left(\left.\mathrm{~d}_{x^{k}} F\right|_{T_{x^{k}} X_{\beta}}\right)=v\left(\mathrm{~d}_{x^{k}}\left(\left.F\right|_{X_{\beta}}\right)\right)=v\left(\mathrm{~d}_{x^{k}}\left(\left.f\right|_{X_{\beta}}\right)\right) \rightarrow$ 0 . Moreover, since $\left.f\right|_{X_{\alpha}}$ is a submersion at $x$, so $F$ is a submersion at $x$. Hence $v\left(\mathrm{~d}_{x}\left(\left.F\right|_{X_{\alpha}}\right)\right) \neq 0$. Since the stratification is Whitney, it implies that $T \supset T_{x} X_{\alpha}$. Consequently $v\left(\left.\mathrm{~d}_{x} F\right|_{T}\right) \geqslant v\left(\mathrm{~d}_{x}\left(\left.F\right|_{X_{\alpha}}\right)\right) \neq 0$. To get a contradiction, we will need the following claim:

Claim 3.8 Let $A_{k}: \mathbb{R}^{q} \rightarrow \mathbb{R}^{p}$ be a sequence of linear maps such that $A_{k} \rightarrow A$ as $k \rightarrow$ $+\infty$ (i.e., the terms of the matrix of $A_{k}$ tend to the corresponding terms of the matrix of $A)$. Let $H_{k} \subset \mathbb{R}^{q}$ be a sequence of linear subspaces of same dimension such that $H_{k} \rightarrow H$ (i.e., $\delta\left(H_{k}, H\right) \rightarrow 0$, where $\delta\left(H_{k}, H\right):=\sup _{y \in H_{k},\|y\|=1} \operatorname{dist}(y, H)$ is the distance between $H_{k}$ and $H$; $\operatorname{dist}(\cdot, \cdot)$ is the Euclidean distance). Then $v\left(\left.A_{k}\right|_{H_{k}}\right) \rightarrow$ $v\left(\left.A\right|_{H}\right)$.

Proof It is clear that

$$
\left\|v\left(\left.A_{k}\right|_{H_{k}}\right)-v\left(\left.A\right|_{H}\right)\right\| \leqslant\left\|v\left(\left.A_{k}\right|_{H_{k}}\right)-v\left(\left.A\right|_{H_{k}}\right)\right\|+\left\|v\left(\left.A\right|_{H_{k}}\right)-v\left(\left.A\right|_{H}\right)\right\| .
$$

In light of [15, Lem. 2.1 (iv)], we have

$$
\left\|v\left(\left.A_{k}\right|_{H_{k}}\right)-v\left(\left.A\right|_{H_{k}}\right)\right\| \leqslant\left\|\left.A_{k}\right|_{H_{k}}-\left.A\right|_{H_{k}}\right\|=\left\|\left.\left(A_{k}-A\right)\right|_{H_{k}}\right\| \leqslant\left\|A_{k}-A\right\| \rightarrow 0 .
$$

Note that $v(A)$ is the length of a minimal semiaxis of $A(B)$, where $B$ is the unit ball. Since $H_{k} \rightarrow H$, we have $V_{k}:=B \cap H_{k} \rightarrow B \cap H:=V$ and $A\left(V_{k}\right) \rightarrow A(V)$ by continuity of $A$. Hence also $v\left(\left.A\right|_{H_{k}}\right) \rightarrow v\left(\left.A\right|_{H}\right)$.

Applying Claim 3.8 with $A_{k}=\mathrm{d}_{x^{k}} F$ and $H_{k}=T_{x^{k}} X_{\beta}$, we get

$$
0=\lim _{k \rightarrow \infty} v\left(\left.\mathrm{~d}_{x^{k}} F\right|_{T_{x^{k}} X_{\beta}}\right)=v\left(\left.\mathrm{~d}_{x} F\right|_{T}\right),
$$

which is a contradiction. The lemma follows. 
For each $\beta \in I^{\prime}$, it is clear that $\left.f\right|_{X_{\beta}}$ is a submersion on $\left(\left.f\right|_{X_{\beta}}\right)^{-1}(\bar{B})$, so for $x \in \bar{U} \cap X_{\beta}$, the differential $\mathrm{d}_{x}\left(\left.f\right|_{X_{\beta}}\right): T_{x} X_{\beta} \rightarrow \mathbb{R}^{2 m}$ is surjective, which induces an isomorphism of vector spaces

$$
\widetilde{\mathrm{d}_{x}}\left(\left.f\right|_{X_{\beta}}\right): T_{x} X_{\beta} / \operatorname{ker} \mathrm{d}_{x}\left(\left.f\right|_{X_{\beta}}\right) \cong \mathbb{R}^{2 m} .
$$

Thus, for each $i=1, \ldots, 2 m$, the vector field $\partial_{i}$ can be lifted uniquely and smoothly on each stratum $X_{\beta}$ with $\beta \in I^{\prime}$ to the vector field called the horizontal lift of $\partial_{i}$ and denoted by $v_{i}^{\beta}$. Clearly, $v_{i}^{\beta}(x)$ is the unique vector in $T_{x} X_{\beta}$ which lifts $\partial_{i}$ and is orthogonal to $\operatorname{ker} \mathrm{d}_{x}\left(\left.f\right|_{X_{\beta}}\right)$. Each $v_{i}^{\beta}$ has the following important properties:

Lemma 3.9 Let $c>0$ be the constant in Lemma 3.7. Then, for each $x \in \bar{U} \cap X_{\beta}$ with $\beta \in I^{\prime}$, we have

$$
\left\|v_{i}^{\beta}(x)\right\| \leqslant \frac{\|x\|+1}{c} .
$$

Proof Let $\mathbb{B}_{\beta}$ be the closed unit ball centered at the origin in $T_{x} X_{\beta}$. Then $\mathrm{d}_{x}\left(\left.f\right|_{X_{\beta}}\right)\left(\mathbb{B}_{\beta}\right)$ is an ellipsoid in $\mathbb{R}^{2 m}$ with $v\left(\mathrm{~d}_{x}\left(\left.f\right|_{X_{\beta}}\right)\right)$ as the length of the shortest semiaxis. Let $\mathbb{B}^{2 m}$ be the closed unit ball centered at the origin in $\mathbb{R}^{2 m}$. Then $\left(\widetilde{\mathrm{d}}_{x}\left(\left.f\right|_{X_{\beta}}\right)\right)^{-1}\left(v\left(\mathrm{~d}_{x}\left(\left.f\right|_{X_{\beta}}\right)\right) \mathbb{B}^{2 m}\right)$ is an ellipsoid in $T_{x} X_{\beta}$ with 1 as the length of the longest semiaxis. Therefore the longest semiaxis of the ellipsoid $\left(\widetilde{\mathrm{d}}_{x}\left(\left.f\right|_{X_{\beta}}\right)\right)^{-1}\left(\mathbb{B}^{2 m}\right)$ is $1 / v\left(\mathrm{~d}_{x}\left(\left.f\right|_{X_{\beta}}\right)\right)$. Consequently,

$$
\left\|v_{i}^{\beta}(x)\right\| \leqslant \frac{1}{v\left(\mathrm{~d}_{x}\left(\left.f\right|_{X_{\beta}}\right)\right)} \leqslant \frac{\|x\|+1}{c},
$$

which yields the lemma.

Note that, for fixed $i$, the vector field on $U$ which coincides with $v_{i}^{\beta}$ on each $U_{\beta}$ is not necessarily a rugose vector field. In what follows, we will try to deform these vector fields to produce a rugose vector field which satisfies the assumption of Lemma 3.5. The process is carried out by induction on dimension.

For $2 m \leqslant d \leqslant 2 \operatorname{dim}_{\mathbb{C}} X$, let $I_{d}^{\prime}:=\left\{\beta \in I^{\prime}: 2 m \leqslant \operatorname{dim} X_{\beta} \leqslant d\right\}$ and $U_{d}:=$ $\cup_{\beta \in I_{d}^{\prime}} X_{\beta} \cap U$. By induction on $d$, we construct a rugose vector field on $U_{2} \operatorname{dim}_{\mathbb{C}} X$ with the property of Lemma 3.5. For $d=2 m$, let $v_{i}^{2 m}$ be the restriction to $U_{2 m}$ of the smooth vector field on $\bigcup_{\beta \in I_{2 m}^{\prime}} X_{\beta}$ which coincides with each $v_{i}^{\beta}$ on $X_{\beta}$ for $\beta \in I_{2 m}^{\prime}$. Then $v_{i}^{2 m}$ is clearly rugose, $\mathrm{d} f\left(v_{i}^{2 m}\right)=\partial_{i}$ and by Lemma $3.9,\left\|v_{i}^{2 m}(x)\right\| \leqslant \frac{\|x\|+1}{c}$ for any $x \in U_{2 m}$.

For each $i$, assume that we have constructed a rugose vector field, denoted by $v_{i}^{d}$, on $U_{d}$ such that $\mathrm{d}_{x} f\left(v_{i}^{d}(x)\right)=\partial_{i}$ and $\left\|v_{i}^{d}(x)\right\| \leqslant \frac{\|x\|+1}{c_{d}}$ for every $x \in U_{d}$, where $c_{d}$ is a positive constant. We need to extend each $v_{i}^{d}$ to a rugose vector field $v_{i}^{d+2}$ on $U_{d+2}$ such that $\left\|v_{i}^{d+2}(x)\right\| \leqslant \frac{\|x\|+1}{c_{d+2}}$ for every $x \in U_{d+2}$, where $c_{d+2}$ is also a positive constant (recall that the strata of $\mathcal{S}$ have even dimension). Note that, to construct $v_{i}^{d+2}$, 
it is enough to construct $v_{i}^{d+2}$ separately on each stratum $X_{\alpha}$ with $\alpha \in I_{d+2}^{\prime} \backslash I_{d}^{\prime}$. Without loss of generality, suppose that $I_{d+2}^{\prime} \backslash I_{d}^{\prime}=\{\alpha\}$. By [20, Lem. 4.4], for each $i=1, \ldots, 2 m$, there is a rugose vector field on $U_{d+2}$, denoted by $\widetilde{w}_{i}^{d+2}$, which extends $v_{i}^{d}$, so the restriction $\left.\widetilde{w}_{i}^{d+2}\right|_{U_{d+2} \cap X_{\alpha}}$ is a smooth vector field. We need to adjust $\widetilde{w}_{i}^{d+2}$ to get a new rugose vector field $w_{i}^{d+2}$ on $U_{d+2}$ such that, for any $y \in X_{\alpha} \cap U_{d+2}$, we have $\mathrm{d}_{y} f\left(w_{i}^{d+2}(y)\right)=\partial_{i}$.

Lemma 3.10 For $y \in U_{d+2} \cap X_{\alpha}$, write

$$
\widetilde{w}_{i}^{d+2}(y)=\sum_{j=1}^{2 m} a_{j}(y) v_{j}^{\alpha}(y)+P(y)
$$

where $P(y) \in \operatorname{ker}_{y} f$. Define

$$
w_{i}^{d+2}(x):= \begin{cases}v_{i}^{\alpha}(x)+P(x) & \text { if } x \in X_{\alpha} \cap U_{d+2} \\ v_{i}^{d}(x) & \text { if } x \in U_{d}\end{cases}
$$

Then $w_{i}^{d+2}$ is a rugose vector field on $U_{d+2}$ and $\mathrm{d}_{x} f\left(w_{i}^{d+2}(x)\right)=\partial_{i}$ for $x \in U_{d+2}$.

Proof For $x^{\prime} \in U_{d}$, let

$$
v_{i}^{d}\left(x^{\prime}\right)=\sum_{j=1}^{2 m} b_{j}\left(x^{\prime}, y\right) v_{j}^{\alpha}(y)+Q\left(x^{\prime}, y\right)+S\left(x^{\prime}, y\right),
$$

where $Q\left(x^{\prime}, y\right) \in \operatorname{kerd}_{y} f$ and $S\left(x^{\prime}, y\right) \in\left(T_{y} X_{\alpha}\right)^{\perp}$. Since $\widetilde{w}_{i}^{d+2}$ is rugose, for each $x \in U_{d}$, there is a neighborhood $W_{x}$ of $x$ such that, for any $y \in W_{x} \cap X_{\alpha}$ and any $x^{\prime} \in W_{x} \cap X_{\beta}$, we have:

- $\left\|P(y)-Q\left(x^{\prime}, y\right)\right\|<C\left\|y-x^{\prime}\right\|$,

- $\left\|S\left(x^{\prime}, y\right)\right\|<C\left\|y-x^{\prime}\right\|$

for some $C>0$, where $X_{\beta}$ is the stratum containing $x$. Shrinking $W_{x}$ and increasing $C$ if necessary, we can suppose that $\mathrm{d} F: x \mapsto \mathrm{d}_{x} F$ is Lipschitz on $W_{x}$, where $F$ is a polynomial extension of $f$ to $\mathbb{C}^{n}$. Hence $\left\|\mathrm{d}_{y} F-\mathrm{d}_{x^{\prime}} F\right\|<C\left\|y-x^{\prime}\right\|$. In particular,

$$
\left\|\mathrm{d}_{y} F\left(v_{i}^{d}\left(x^{\prime}\right)\right)-\mathrm{d}_{x^{\prime}} F\left(v_{i}^{d}\left(x^{\prime}\right)\right)\right\|<C\left\|y-x^{\prime}\right\| \cdot\left\|v_{i}^{d}\left(x^{\prime}\right)\right\|,
$$

i.e.,

$$
\left\|\sum_{j=1}^{2 m} b_{j}\left(x^{\prime}, y\right) \partial_{j}-\partial_{i}\right\|-\left\|\mathrm{d}_{y} F\left(S\left(x^{\prime}, y\right)\right)\right\|<C\left\|y-x^{\prime}\right\| \cdot\left\|v_{i}^{d}\left(x^{\prime}\right)\right\| .
$$


Shrink $W_{x}$, if necessary, so that $\bar{W}_{x} \cap X_{\gamma} \subset U_{d+2}$ for any $\gamma \in I_{d+2}^{\prime}$. Let $M:=$ $\sup _{z \in U_{d}}\left\|v_{i}^{d}\right\|$ and $N:=\sup _{z \in W_{x}}\left\|\mathrm{~d}_{z} F\right\|$. Then we have

$$
\sum_{j \neq i}\left|b_{j}\left(x^{\prime}, y\right)\right|+\left|b_{i}\left(x^{\prime}, y\right)-1\right|<C(M+N)\left\|y-x^{\prime}\right\|
$$

and

$$
\begin{aligned}
\left\|w_{i}^{d+2}(y)-v_{i}^{d}\left(x^{\prime}\right)\right\|< & \left(\sum_{j \neq i}\left|b_{j}\left(x^{\prime}, y\right)\right|+\left|b_{i}\left(x^{\prime}, y\right)-1\right|\right) D \\
& +\left\|P(y)-Q\left(x^{\prime}, y\right)\right\|+\left\|S\left(x^{\prime}, y\right)\right\|,
\end{aligned}
$$

where $D:=\sup _{z \in W_{x} \cap X_{\alpha}}\left\|v_{i}^{\alpha}\right\|$. Thus

$$
\left\|w_{i}^{d+2}(y)-v_{i}^{d}\left(x^{\prime}\right)\right\|<(2 C+C D(M+N))\left\|y-x^{\prime}\right\| .
$$

Hence $w_{i}^{d+2}$ is rugose and of course $\mathrm{d}_{x} f\left(w_{i}^{d+2}(x)\right)=\partial_{i}$ for $x \in U_{d+2}$.

Since $w_{i}^{d+2}$ is rugose (in view of Lemma 3.10), it is continuous. Then, by shrinking $W_{x}$, where $W_{x}$ is determined in the proof of Lemma 3.10, if necessary, we may assume that $W_{x} \subset\left\{z \in \mathbb{C}^{n}:\|z-x\| \leqslant 1\right\}$ and

$$
\left\|w_{i}^{d+2}(y)\right\|<2\left\|v_{i}^{d}(x)\right\|
$$

for any $y \in W_{x} \cap X_{\alpha}$. Let $W_{d}:=\bigcup_{x \in U_{d}} W_{x}$, then $W_{d}$ is an open neighborhood of radius no larger than 1 of $U_{d}$. Let $F=\left(f_{1}, \ldots, f_{m}\right): \mathbb{C}^{n} \rightarrow \mathbb{C}^{m}$ be the polynomial extending $f$ on $\mathbb{C}^{n}$. Let $K:=F^{-1}(B)$, which is considered as a subset of $\mathbb{R}^{2 n}$ under the identification of $\mathbb{C}^{n}$ with $\mathbb{R}^{2 m}$. Then $U_{d}$ is a closed set in $K$ for the induced topology from the Euclidean topology. By a smooth version of Urysohn's lemma, there is a smooth function $\varphi: K \rightarrow[0,1]$ such that $\varphi^{-1}(0)=K \backslash W_{d}$ and $\varphi^{-1}(1)=U_{d}$. (Note that there may not exist such a function $\varphi$ defined on the whole of $\mathbb{R}^{2 n}$ since $U_{d}$ is not closed in $\mathbb{R}^{2 n}$; namely, there may be no smooth extensions of $\varphi$ on $\mathbb{R}^{2 n}$.) For $x \in U_{d+2}$, set

$$
v_{i}^{d+2}(x):=(1-\varphi(x)) v_{i}^{\alpha}(x)+\varphi(x) w_{i}^{d+2}(x) .
$$

Clearly, the restriction of $v_{i}^{d+2}$ on each stratum is a smooth vector field. Moreover, we have

$$
\begin{aligned}
\mathrm{d}_{x} f\left(v_{i}^{d+2}(x)\right) & =\mathrm{d}_{x} f\left((1-\varphi(x)) v_{i}^{\alpha}(x)+\varphi(x) w_{i}^{d+2}(x)\right) \\
& =(1-\varphi(x)) \mathrm{d}_{x} f\left(v_{i}^{\alpha}(x)\right)+\varphi(x) \mathrm{d}_{x} f\left(w_{i}^{d+2}(x)\right) \\
& =(1-\varphi(x)) \partial_{i}+\varphi(x) \partial_{i}=\partial_{i} .
\end{aligned}
$$


Let us prove that $v_{i}^{d+2}$ is a rugose vector field. For any $x \in U_{d}$, let $X_{\beta}$ be the stratum containing $x$. For $x^{\prime} \in W_{x} \cap X_{\beta}$ and $y \in W_{x} \cap X_{\alpha}$, we have

$$
\begin{aligned}
& \left\|v_{i}^{d+2}(y)-v_{i}^{d+2}\left(x^{\prime}\right)\right\| \\
& \quad=\left\|(1-\varphi(y)) v_{i}^{\alpha}(y)+\varphi(y) w_{i}^{d+2}(y)-v_{i}^{d}\left(x^{\prime}\right)\right\| \\
& \quad=\left\|(1-\varphi(y)) v_{i}^{\alpha}(y)-(1-\varphi(y)) w_{i}^{d+2}(y)+w_{i}^{d+2}(y)-v_{i}^{d}\left(x^{\prime}\right)\right\| \\
& \quad \leqslant(1-\varphi(y))\left\|v_{i}^{\alpha}(y)-w_{i}^{d+2}(y)\right\|+\left\|w_{i}^{d+2}(y)-v_{i}^{d}\left(x^{\prime}\right)\right\| \\
& \quad \leqslant(1-\varphi(y))\left(\left\|v_{i}^{\alpha}(y)\right\|+\left\|w_{i}^{d+2}(y)\right\|\right)+\left\|w_{i}^{d+2}(y)-w_{i}^{d+2}\left(x^{\prime}\right)\right\| .
\end{aligned}
$$

We note the following facts:

- Since $1-\varphi(y)$ is a smooth function, it is locally Lipschitz; with no loss of generality, assume that $1-\varphi(y)$ is Lipschitz on $W_{x}$ with constant $c_{1}$. Then

$$
1-\varphi(y)=(1-\varphi(y))-\left(1-\varphi\left(x^{\prime}\right)\right) \leqslant c_{1}\left\|y-x^{\prime}\right\| .
$$

- By Lemma 3.9 and by the continuity of $w_{i}^{d+2}$, there is a positive constant $c_{2}$ depending only on $x$ such that $\left\|v_{i}^{\alpha}(y)\right\|+\left\|w_{i}^{d+2}(y)\right\| \leqslant c_{2}$ (we can take $c_{2}:=$ $\sup _{z \in \bar{W}_{x} \cap X_{\alpha}} \frac{\|z\|+1}{c}+\sup _{z \in \bar{W}_{x} \cap X_{\alpha}}\left\|w_{i}^{d+2}(z)\right\|$; note that $\left.\bar{W}_{x} \cap X_{\alpha} \subset U_{d+2}\right)$.

- Since $w_{i}^{d+2}$ is rugose, it follows that there is a positive constant $c_{3}$ depending only on $x$ such that $\left\|w_{i}^{d+2}(y)-w_{i}^{d+2}\left(x^{\prime}\right)\right\| \leqslant c_{3}\left\|y-x^{\prime}\right\|$.

Hence

$$
\left\|v_{i}^{d+2}(y)-v_{i}^{d+2}\left(x^{\prime}\right)\right\| \leqslant\left(c_{1} c_{2}+c_{3}\right)\left\|y-x^{\prime}\right\|,
$$

i.e., $v_{i}^{d+2}$ is rugose. Now it remains to show that there is a positive constant $c_{d+2}$ such that $\left\|v_{i}^{d+2}(y)\right\| \leqslant \frac{\|y\|+1}{c_{d+2}}$ for every $y \in U_{d+2}$. Obviously, the statement holds for $y \in U_{d}$ by the induction assumption and for $y \in U_{d+2} \backslash W_{d}$ by Lemma 3.9, so we can suppose that $y \in W \backslash U_{d}$, which clearly implies that $y \in X_{\alpha}$. In light of Lemma 3.9, we get

$$
\left\|v_{i}^{\alpha}(y)\right\| \leqslant \frac{\|y\|+1}{c},
$$

where $c$ is the constant in the same lemma. In view of (4) and the induction assumption, we have

$$
\begin{aligned}
\left\|w_{i}^{d+2}(y)\right\| & <2\left\|v_{i}^{d}(x)\right\| \\
& \leqslant 2 \frac{\|x\|+1}{c_{d}} \leqslant 2 \frac{\|y\|+\|x-y\|+1}{c_{d}} \leqslant 2 \frac{\|y\|+2}{c_{d}} \leqslant 4 \frac{\|y\|+1}{c_{d}} .
\end{aligned}
$$


Thus (5) and (6) yield

$$
\begin{aligned}
\left\|v_{i}^{d+2}(y)\right\| & =\left\|(1-\varphi(y)) v_{i}^{\alpha}(y)+\varphi(y) w_{i}^{d+2}(y)\right\| \\
& \leqslant(1-\varphi(y))\left\|v_{i}^{\alpha}(y)\right\|+\varphi(y)\left\|w_{i}^{d+2}(y)\right\| \\
& \leqslant(1-\varphi(y)) \frac{\|y\|+1}{c}+\varphi(y) 4 \frac{\|y\|+1}{c_{d}} \\
& <\left(\frac{1}{c}+\frac{4}{c_{d}}\right)(\|y\|+1) .
\end{aligned}
$$

Set $c_{d+2}=\min \left\{\frac{1}{1 / c+4 / c_{d}}, c, c_{d}\right\}$, then $\left\|v_{i}^{d+2}(y)\right\| \leqslant \frac{\|y\|+1}{c_{d+2}}$ for every $y \in U_{d+2}$. By induction, there exists a rugose vector field on $U_{2} \operatorname{dim}_{\mathbb{C}} X$ with the property of Lemma 3.5. Then the theorem follows by applying Lemma 3.5.

The following corollary follows immediately from Theorem 3.4:

Corollary 3.11 Let $X \subset \mathbb{C}^{n}$ be an affine variety with an affine Whitney stratification $\mathcal{S}$, and let $f: X \rightarrow \mathbb{C}^{m}$ be a polynomial dominant map. Assume that, for any stratum $X_{\beta} \in \mathcal{S}$, the restriction $\left.f\right|_{X_{\beta}}$ is a submersion and $K_{\infty}\left(f, X_{\beta}\right)=\emptyset$. Then $f$ is a locally trivial fibration.

\section{Computation of the Sets of Stratified Generalized Critical Values}

In this section, we compute the set $K(f, \mathcal{S})$ of stratified generalized critical values of $f$, for which we need to construct an affine Whitney stratification of $X$ and then apply [10] for each stratum of this stratification. The process is slightly different from the construction in Sect. 2.3, since we only need to construct such an affine Whitney stratification "partially," by noting the following facts:

- As the construction of Whitney stratifications is by induction on dimension, we only need to proceed until the dimension shrinks below $m$, since the restriction of $f$ to any stratum of dimension $<m$ is always singular.

- For any algebraic set $Z \subseteq X$, let

$$
\begin{aligned}
& r_{Z}:=\max _{x \in Z \backslash V\left(p_{Z, \emptyset}\right)} \operatorname{rank} \operatorname{Jac}_{x}\left(\left.f\right|_{Z}\right) \\
& \quad \text { and } H(Z):=\overline{\left\{x \in Z \backslash V\left(p_{Z, \emptyset}\right): \operatorname{rank} \operatorname{Jac}_{x}\left(\left.f\right|_{Z}\right)<r_{Z}\right\}} \mathcal{Z} .
\end{aligned}
$$

Then, at any step of the induction process, the construction in Sect. 2.3 can be omitted if $r_{Y}<m$.

Let us now construct such a stratification. With the same notations as in Lemma 2.8, let 


$$
\Gamma_{3}:=\bigcup_{k=1}^{t}\left\{\begin{array}{l}
(x, x, w, v, \gamma, \lambda, \mu) \in \mathbb{C}^{n} \times \mathbb{C}^{n} \times \mathbb{C}^{n} \times \mathbb{C}^{n} \times \mathbb{C} \times \mathbb{C} \times \mathbb{C}^{t}: \\
h_{1}(x, x, w, v)=\cdots=h_{q}(x, x, w, v)=0 \\
\gamma \sum_{j=1}^{n} v_{j} w_{j}=1 \\
\lambda p_{Y, \emptyset}(x)=1 \\
\mu_{k} M_{k}^{(m, p)}(x)=1
\end{array}\right\},
$$

where each $M_{k}^{(m, p)}(x)$ is a minor of the matrix

$$
A(x):=\left[\begin{array}{c}
\mathrm{d}_{x} f_{1} \\
\vdots \\
\mathrm{d}_{x} f_{m} \\
\mathrm{~d}_{x} g_{1} \\
\vdots \\
\mathrm{d}_{x} g_{r} \\
\mathrm{~d}_{x} \widetilde{g}_{r+1} \\
\vdots \\
\mathrm{d}_{x} \widetilde{g}_{p}
\end{array}\right],
$$

obtained by deleting $n-m-p$ columns. So $\Gamma_{3}$ differs from $\Gamma_{2}$ in the last $t$ equations, since we are only interested in finding the points where the Whitney condition (b) is not satisfied, outside $P(Y, \emptyset)$. Let

$$
\pi_{3}: \mathbb{C}^{n} \times \mathbb{C}^{n} \times \mathbb{C}^{n} \times \mathbb{C}^{n} \times \mathbb{C} \times \mathbb{C} \times \mathbb{C}^{t} \rightarrow \mathbb{C}^{n}
$$

be the projection on the first $n$ coordinates. By Lemma 2.8, $\pi_{3}\left(\Gamma_{3}\right)$ is the set of points where the Whitney condition (b) fails. Obviously $\pi_{3}\left(\Gamma_{3}\right) \subset \operatorname{reg}\left(\left.f\right|_{Y \backslash P(Y)}\right)$ and $\operatorname{dim} \pi_{3}\left(\Gamma_{3}\right)<\operatorname{dim} Y$. Set $\widetilde{W}:=\overline{\pi_{3}\left(\Gamma_{3}\right)} \mathcal{Z}$. Then obviously $\operatorname{dim} \widetilde{W}<\operatorname{dim} Y$. Again, we can compute a Gröbner basis of the ideal $I(\widetilde{W})$. Finally, set

- $X_{0}:=X$,

- $X_{1}:=X_{0} \cap V\left(p_{\left.X_{0}, \emptyset\right)}\right), S_{1}=K_{0}\left(f, X_{0} \backslash X_{1}\right), \ldots$,

- $X_{i}:=X_{i-1} \cap V\left(p_{X_{i-1}, \bigcup_{j=0}^{i-2} \widetilde{W}\left(X_{j}, X_{i-1}\right)}\right), S_{i}=K_{0}\left(f, X_{i-1} \backslash X_{i}\right), \ldots$

By induction, we can construct a finite filtration of algebraic sets $X=X_{0} \supset X_{1} \supset$ $\cdots \supset X_{q} \supset X_{q+1} \supseteq \emptyset$ with $\operatorname{dim} X_{i}>\operatorname{dim} X_{i+1}$ and $r_{X_{q+1}}<m$. It is clear that this filtration does not induce an affine Whitney stratification $\mathcal{S}$ of $X$. However, it shows that there is an affine Whitney stratification $\mathcal{S}$ such that

$$
K_{0}(f, \mathcal{S})=\bigcup_{i=1}^{q} S_{i} \cup \overline{f\left(X_{q+1}\right)}
$$

Let $Z_{i}:=X_{i} \backslash X_{i+1}$. Then $\left\{Z_{i}\right\}_{i=0, \ldots, q}$ is an affine Whitney stratification of $X \backslash X_{q+1}$. Every variety $Z_{i}$ can be realized as a closed affine variety $\widetilde{Z}_{i}$ in $\mathbb{C}^{n+1}$, by the embedding $Z_{i} \ni x \mapsto\left(x, 1 / P_{X_{i}, \bigcup_{j=0}^{i-1} \widetilde{W}\left(X_{j}, X_{i}\right)}(x)\right) \in \mathbb{C}^{n+1}$ for $i>0$ or the embedding $Z_{0} \ni$ 
$x \mapsto\left(x, 1 / P_{X_{0}, \emptyset}(x)\right) \in \mathbb{C}^{n+1}$. Let $K_{\infty}\left(f, Z_{i}\right)$ be the set of asymptotic critical values of $\left.f\right|_{Z_{i}}$, which now can be computed analogously as in $[8,10]$ - this will be done in the next section. Then, from the construction, it is clear that the set of stratified generalized critical values of $f$ is given by

$$
K(f, \mathcal{S}):=\bigcup_{i=1}^{q}\left(K_{\infty}\left(f, Z_{i}\right) \cup K_{0}\left(f, Z_{i}\right)\right) \cup \overline{f\left(X_{q+1}\right)}
$$

and $K(f, \mathcal{S})$ can be computed effectively. Note that Remark 2.5 and elementary properties of Gröbner bases imply:

Corollary 4.1 Let $X \subset \mathbb{C}^{n}$ be an affine variety of pure dimension and let $f=$ $\left(f_{1}, \ldots, f_{m}\right): X \rightarrow \mathbb{C}^{m}$ be a polynomial mapping. Let $\mathbb{F} \subset \mathbb{C}$ be a subfield generated by coefficients of generators of $I(X)$ and all coefficients of polynomials $f_{i}, i=1, \ldots, m$. Then there is a nowhere dense affine variety $K(f, \mathcal{S}) \subset \mathbb{C}^{m}$, which is described by polynomials from $\mathbb{F}\left[x_{1}, \ldots, x_{m}\right]$ such that all bifurcation values $B(f)$ of $f$ are contained in $K(f, \mathcal{S})$. In particular, for $m=1$, if $X$ and $f$ are described by polynomials from $\mathbb{Q}\left[x_{1}, \ldots, x_{n}\right]$, then all bifurcation values of $f$ are algebraic numbers.

\section{Computation of $K_{0}\left(f, Z_{i}\right) \cup K_{\infty}\left(f, Z_{i}\right)$}

Let $k=\mathbb{R}$ or $k=\mathbb{C}$. Let $X \cong k^{n}, Y \cong k^{m}$ be finite-dimensional vector spaces (over $k$ ). We consider those spaces equipped with the canonical scalar (hermitian) products. Let us denote by $\mathcal{L}(X, Y)$ the set of linear mappings from $X$ to $Y$ and by $\Sigma=\Sigma(X, Y) \subset \mathcal{L}(X, Y)$ the set of nonsurjective mappings. In this section, we give several different expressions for a distance of an $A \in \mathcal{L}(X, Y)$ to the space $\Sigma$ of singular operators. Let us first recall the following [15]:

Definition 5.1 Let $A \in \mathcal{L}(X, Y)$. Set

$$
v(A)=\inf _{\|\phi\|=1}\left\|A^{*}(\phi)\right\|,
$$

where $A^{*} \in \mathcal{L}\left(Y^{*}, X^{*}\right)$ is the adjoint operator and $\phi \in Y^{*}$.

Let $\alpha, \beta: \mathcal{L}(X, Y) \rightarrow \mathbb{R}_{+}$be two nonnegative functions. We shall say that $\alpha$ and $\beta$ are equivalent (we write $\alpha \sim \beta$ ) if there are constants $c, d>0$ such that

$$
c \alpha(A) \leq \beta(A) \leq d \alpha(A)
$$

for any $A \in \mathcal{L}(X, Y)$. We give below several functions equivalent to $v$. Let $A=$ $\left(A_{1}, \ldots, A_{m}\right) \in \mathcal{L}(X, Y)$, and let $\overline{A_{i}}=\operatorname{grad} A_{i}$. Denote by $\left\langle\left(\overline{A_{j}}\right)_{j \neq i}\right\rangle$ the linear space generated by vectors $\left(\overline{A_{j}}\right), j \neq i$. Let 


$$
\kappa(A)=\min _{1 \leq i \leq m} \operatorname{dist}\left(\overline{A_{i}},\left\langle\left(\overline{A_{j}}\right)_{j \neq i}\right\rangle\right)
$$

be the Kuo number of $A$.

Proposition 5.2 ([12]) The Kuo function $\kappa$ is equivalent to the $v$ of Rabier. More precisely,

$$
v(A) \leq \kappa(A) \leq \sqrt{m} v(A) .
$$

Definition 5.3 Let $A \in \mathcal{L}(X, Y)$, and let $H \subset X$ be a linear subspace. We set

$$
v(A, H)=v\left(\left.A\right|_{H}\right), \quad \kappa(A, H)=\kappa\left(\left.A\right|_{H}\right),
$$

where $\left.A\right|_{H}$ denotes the restriction of $A$ to $H$.

From Proposition 5.2 we immediately get the following corollary:

Corollary 5.4 We have $v(A, H) \sim \kappa(A, H)$.

In fact we also have the following explicit expression for $\kappa(A, H)($ see $[9,10])$ :

Proposition 5.5 Let $A=\left(A_{1}, \ldots, A_{m}\right) \in \mathcal{L}(X, Y)$, and let $H \subset X$ be a linear subspace. Assume that $H$ is given by a system of linear equations $B_{j}=0, j=$ $1, \ldots, r$. Then

$$
\kappa(A, H)=\min _{1 \leq i \leq m} \operatorname{dist}\left(\overline{A_{i}},\left\langle\left(\overline{A_{j}}\right)_{j \neq i} ;\left(\overline{B_{j}}\right)_{j=1, \ldots, r}\right\rangle\right),
$$

where $\overline{A_{i}}=\operatorname{grad} A_{i}$ and $\overline{B_{j}}=\operatorname{grad} B_{j}$.

Finally, we introduce a function $g^{\prime}$ which will be useful in the explicit description of the set of generalized critical values.

Definition 5.6 Let $A \in \mathcal{L}\left(k^{n}, k^{m}\right)$, where $n \geq m+r$, and let $H \subset k^{n}$ be a linear subspace given by a system of independent linear equations $B_{l}=\sum b_{l k} x_{k}, l=$ $1, \ldots, r$. By abuse of notation, we denote by $A$ the matrix (in the canonical bases in $k^{n}$ and $\left.k^{m}\right)$ of the mapping $A$. Let $C$ be an $(m+r) \times n$ matrix given by rows $A_{1}, \ldots, A_{m} ; B_{1}, \ldots, B_{r}$ (we identify $A_{i}=\sum a_{i k} x_{k}$ with the vector $\left(a_{j 1}, \ldots, a_{j n}\right)$, similarly for $\left.B_{l}\right)$. Let $M_{I}$, where $I=\left(i_{1}, \ldots, i_{m+r}\right)$, denote an $(m+r) \times(m+r)$ minor of $C$ given by columns indexed by $I$. Let $M_{J}(j)$ denote an $(m+r-1) \times(m+r-1)$ minor given by columns indexed by $J$ and by deleting the $j^{\text {th }}$ row, where $1 \leq j \leq m$. Note that we delete only $A_{j}$ rows. We set

$$
g^{\prime}(A, H)=\max _{I}\left\{\min _{\{J \subset I, 1 \leq j \leq m\}} \frac{\left|M_{I}\right|}{\left|M_{J}(j)\right|}\right\}
$$

(where we consider only numbers with $M_{J}(j) \neq 0$; if all numbers $M_{J}(j)$ are zero, we put $\left.g^{\prime}(A)=0\right)$. 
In particular, we have the following (see $[9,10])$ :

Proposition 5.7 We have $g^{\prime}(A, H) \sim v(A, H)$.

Now we can prove the following theorem:

Theorem 5.8 Let $Z_{i}$ be a stratum of $X$ as in Sect. 4. Then the set $K\left(f, Z_{i}\right)=$ $K_{0}\left(f, Z_{i}\right) \cup K_{\infty}\left(f, Z_{i}\right)$ is a nowhere dense algebraic subset of $\mathbb{C}^{m}$.

Proof It is a standard fact that $K_{0}\left(f, Z_{i}\right)$ is algebraic and nowhere dense (for details see the end of Sect. 5.1). Hence, it is enough to focus on $K_{\infty}\left(f, Z_{i}\right)$.

By construction, the set $X:=Z_{i} \subset \mathbb{C}^{n}$ is a subset of complete intersection, $X \subset\left\{b_{1}=0, \ldots, b_{s}=0\right\}$, and $\operatorname{rank}\left\{\nabla b_{k}: k=1, \ldots, s\right\}=s(X$ has codimension $s)$. Let us recall notation of Definition 5.6. For $x \in \mathbb{C}$, let $A=\mathrm{d}_{x} f$ and $B_{l}=\mathrm{d}_{x} b_{l}$, $l=1, \ldots, s$. Let $A \in \mathcal{L}\left(k^{n}, k^{m}\right)$, where $n \geq m+s$, and let $T_{x} X=H \subset k^{n}$ be a linear subspace given by a system of independent linear equations $B_{l}=\sum b_{l k} x_{k}, l=$ $1, \ldots, s$. By abuse of notation, we denote by $A$ the matrix (in the canonical bases in $k^{n}$ and $\left.k^{m}\right)$ of the mapping $A$. Let $C$ be an $(m+s) \times n$ matrix given by rows $A_{1}, \ldots, A_{m} ; B_{1}, \ldots, B_{s}$ (we identify $A_{i}=\sum a_{i k} x_{k}$ with the vector $\left(a_{j 1}, \ldots, a_{j n}\right)$, similarly for $B_{l}$ ).

For an index $I=\left(i_{1}, \ldots, i_{m+r}\right) \subset\{1, \ldots, n\}$ let $M_{I}(x)$ denote the $(m+s) \times(m+s)$ minor of $C$ given by columns indexed by $I$. For integers $j \in I, 1 \leq k \leq m$ we denote by $M_{I(k, j)}(x)$ the $(m+s-1) \times(m+s-1)$ minor obtained by deleting the $j^{\text {th }}$ column and the $k^{\text {th }}$ row. Note that we delete only $A_{k}, 1 \leq k \leq m$ rows.

Hence, $M_{I}$ and $M_{I(k, j)}$ are regular (restriction of polynomials) functions on $X$. We now define a family of rational functions on $X$ :

$$
W_{I(k, j)}(x)=M_{I}(x) / M_{I(k, j)}(x),
$$

where, for $M_{I(k, j)} \equiv 0$, we put $W_{I(k, j)} \equiv 0$. We write $b=\left(b_{1}, \ldots, b_{s}\right)$ and $(f, b): \mathbb{C}^{n} \rightarrow \mathbb{C}^{m} \times \mathbb{C}^{s}$; here, we consider $f_{1}, \ldots, f_{m}$ and $b_{1}, \ldots, b_{s}$ as polynomials on $\mathbb{C}^{n}$.

Let $w=\left(\begin{array}{c}n \\ m+s\end{array}\right)$ and let $M_{I_{1}}, \ldots, M_{I_{w}}$ be all possible main minors of a matrix of $\mathrm{d}_{x}(f, b)$. For every index $I_{l}$, take a pair $\left(k_{l}, j_{l}\right)$ which determines an $(m+s-1) \times$ $(m+s-1)$ minor of $M_{I_{l}}$ (we consider here only minors which are not identically zero). We denote a sequence $\left(k_{1}, j_{1}\right), \ldots,\left(k_{w}, j_{w}\right)$ by $(k, j) \in \mathbb{N}^{w} \times \mathbb{N}^{w}$, and we consider a rational function

$$
\Phi_{(k, j)}=\Phi\left(\left(k_{1}, j_{1}\right), \ldots,\left(k_{w}, j_{w}\right)\right): X \rightarrow \mathbb{C}^{m} \times \mathbb{C}^{N}
$$

where the first component of $\Phi_{(k, j)}$ is $f$ and the next components are $W_{I_{p}\left(k_{p}, j_{p}\right)}$, $p=1, \ldots, w$, and all products $x_{l} W_{I_{p}\left(k_{p}, j_{p}\right)}, p=1, \ldots, w ; l=1, \ldots, n$.

We can assume that, for some choice of $l$, we have $W_{I_{l}\left(k_{l}, j_{l}\right)} \neq \equiv 0$, and consequently $\operatorname{dim} \operatorname{cl}\left(\Phi_{(k, j)}(X)\right)=\operatorname{dim} X=n-s$. Here $\operatorname{cl}(Y)$ stands for the closure of $Y$ in the strong (or which is the same, in the Zariski) topology. Let $\Gamma(k, j)=\operatorname{cl}\left(\Phi_{(k, j)}(X)\right.$ ) (by $\Phi_{(k, j)}(X)$ we mean the set $\Phi_{(k, j)}(X \backslash P)$, where $P$ is a set of poles of $\left.\Phi_{(k, j)}\right)$. 
Now, for a given $r \in\{1, \ldots, n\}$, consider the set $X_{r}:=X \backslash\left\{x_{r}=0\right\}$. Finally, let $\Phi_{(k, j), r}(x):=\left(\Phi_{(k, j)}(x), 1 / x_{r}\right)$ and $\Gamma((k, j), r):=\operatorname{cl}\left(\Phi_{(k, j), r}\left(X_{r}\right)\right)$. Let us recall that $y \in K_{\infty}\left(f, Z_{i}\right)$ if there exists a sequence $x \rightarrow \infty ; x \in Z_{i}$ such that

$$
f(x) \rightarrow y \text { and }\|x\| g^{\prime}(x) \rightarrow 0
$$

where $g^{\prime}(x)=g^{\prime}\left(\mathrm{d}_{x} f, T_{x} Z_{i}\right)$. We have

\section{Lemma 5.9}

$$
K_{\infty}(f, X)=\mathbb{C}^{m} \cap \bigcup_{(k, j), r} \Gamma((k, j), r)
$$

where we identify $\mathbb{C}^{m}$ with $\mathbb{C}^{m} \times(0, \ldots, 0)$.

Proof We identify $X$ with $\widetilde{Z}_{i} \subset \mathbb{C}^{n+1}$, hence we can assume that $X$ is closed in $\mathbb{C}^{n+1}$. Let $y \in K_{\infty}(f, X)$. Hence, there is a sequence $x^{l} \rightarrow \infty$ such that $x^{l} \in X$ and $f\left(x^{l}\right) \rightarrow y$ and $\left\|x^{l}\right\| g^{\prime}\left(x_{l}\right) \rightarrow 0$. Moreover, if $x=\left(x_{1}, \ldots, x_{n}\right)$, then there is at least one $r, 1 \leq r \leq n$, such that $x_{r}^{l} \rightarrow \infty$. If $\left\{x^{l}: l=1,2, \ldots\right\} \subset C(f, X)(C(f, X)$ denotes the set of critical points of $\left.f_{\mid X}\right)$, then it is easy to see that $y \in \mathbb{C}^{m} \cap \Gamma((k, j), r)$ for every $(k, j)$ (we can choose a close sequence $x^{\prime l}$ such that $f\left(x^{\prime l}\right) \rightarrow y$ and $\left\|x^{\prime l}\right\| g^{\prime}\left(x^{\prime l}\right) \rightarrow 0$ and functions $W_{I(k, j)}$ are defined). Consequently, we can assume that $\left\{x^{l}: l=1,2, \ldots\right\} \cap C(f, X)=\emptyset$. Thus, there is a sequence $x^{l} \rightarrow \infty$ such that, for every $I_{i}$, there are integers $\left(k_{i}, j_{i}\right)$ such that $\left\|x^{l}\right\| M_{I_{i}} / M_{I_{i}\left(k_{i}, j_{i}\right)}\left(x_{l}\right) \rightarrow 0$ and $f\left(x^{l}\right) \rightarrow$ $y$. This also gives $y \in \Gamma((k, j), r) \cap \mathbb{C}^{m}$ with $((k, j), r)=\left(\left(k_{1}, j_{1}\right), \ldots,\left(k_{w}, j_{w}\right), r\right)$.

Conversely, if $y \in \Gamma((k, j), r) \cap \mathbb{C}^{m}$, then we can choose a sequence $x^{l} \rightarrow \infty$, $x^{l} \in X_{r}$ such that $f\left(x^{l}\right) \rightarrow y$ and $\left\|x^{l}\right\| M_{I_{i}} / M_{I_{i}\left(k_{i}, j_{i}\right)}\left(x^{l}\right) \rightarrow 0$. It is easy to observe that this implies $\left\|x^{l}\right\| g^{\prime}\left(x^{l}\right) \rightarrow 0$ and $f\left(x^{l}\right) \rightarrow y$, i.e. $y \in K_{\infty}(f, X)$.

Now, in light of [10, Thm. 3.3], we have that $K_{\infty}(f, X) \neq \mathbb{C}^{m}$ hence $\mathbb{C}^{m} \cap$ $\bigcup_{((k, j), r)} \Gamma((k, j), r) \neq \mathbb{C}^{m}$. By Lemma $5.9, K_{\infty}(f, X)$ is an algebraic set. The theorem follows.

\subsection{A Sketch of an Algorithm}

Let $X:=Z_{i} \subset \mathbb{C}^{n}$ be a smooth affine variety of dimension $n-s$. Let $f=$ $\left(f_{1}, \ldots, f_{m}\right): X \rightarrow \mathbb{C}^{m}$ be a polynomial dominant mapping. Then the set $K_{\infty}(f, X)$ can be computed as follows:

By construction, $X$ is a subset of complete intersection, hence we can choose polynomials $b_{1}, \ldots, b_{s} \in I(X)$ such that $\operatorname{rank}\left\{\operatorname{grad} b_{1}, \ldots, \operatorname{grad} b_{s}\right\}=s$ on $X$. Let us consider the rational mapping

$$
\begin{aligned}
& \Phi\left(\left(k_{1}, j_{1}\right), \ldots,\left(k_{w}, j_{w}\right), r\right): X \ni x \\
& \mapsto\left(f(x), W_{I_{1}\left(k_{1}, j_{1}\right)}(x), x_{1} W_{I_{1}\left(k_{1}, j_{1}\right)}(x), \ldots, x_{n} W_{I_{1}\left(k_{1}, j_{1}\right)}(x),\right. \\
& \left.\quad \ldots, W_{I_{s}\left(k_{w}, j_{w}\right)}(x), x_{1} W_{I_{w}\left(k_{w}, j_{w}\right)}(x), \ldots, x_{n} W_{I_{w}\left(k_{w}, j_{w}\right)}(x), 1 / x_{r}\right) \in \mathbb{C}^{m} \times \mathbb{C}^{N},
\end{aligned}
$$


which is constructed exactly as in the proof of Theorem 5.8. Recall that

$$
\Gamma\left(\left(k_{1}, j_{1}\right), \ldots,\left(k_{w}, j_{w}\right), r\right)=\operatorname{cl}\left(\Phi\left(\left(k_{1}, j_{1}\right), \ldots,\left(k_{w}, j_{w}\right), r\right)(X)\right)
$$

We know also that

$$
K_{\infty}(f, X)=L \cap\left(\bigcup_{\left(\left(k_{1}, j_{1}\right), \ldots,\left(k_{w}, j_{w}\right)\right), r} \Gamma\left(\left(k_{1}, j_{1}\right), \ldots,\left(k_{w}, j_{w}\right), r\right)\right),
$$

where $L=\mathbb{C}^{m} \times(0, \ldots, 0)$. First we compute the ideal of the set $\Gamma\left(\left(k_{1}, j_{1}\right), \ldots\right.$, $\left.\left(k_{w}, j_{w}\right), r\right)$. Now we can consider a variety $X$ as a closed affine variety in $\mathbb{C}^{n+1}$ (see the end of Sect. 4). Let $I(X)=\left(b_{1}, \ldots, b_{q}\right)$. To this end, we restrict the mapping $\Phi((k, j), r)$ to an open dense subset $U \subset X$ on which this mapping is regular. In particular, we can choose the set $U=X \backslash\left(\bigcup_{l=1}^{w}\left\{M_{I_{l}\left(k_{l}, j_{l}\right)}=0\right\} \cup\left\{x_{r}=0\right\}\right)$. The set $U$ can be identified with the set

$$
\begin{aligned}
& V\left(\left(k_{1}, j_{1}\right), \ldots,\left(k_{w}, j_{w}\right), r\right) \\
& :=\left\{\left(x, t, z_{1}, \ldots, z_{w}\right) \in X \times \mathbb{C} \times \mathbb{C}^{w}: j=1, \ldots, w ; x_{r} t=1 ;\right. \\
& \left.\quad M_{I_{p}\left(k_{p}, j_{p}\right)} z_{p}=1 ; p=1, \ldots, w\right\} .
\end{aligned}
$$

Now we can consider a morphism

$$
\Psi\left(\left(k_{1}, j_{1}\right), \ldots,\left(k_{w}, j_{w}\right)\right): V\left(\left(k_{1}, j_{1}\right), \ldots,\left(k_{p}, j_{p}\right), r\right) \rightarrow \mathbb{C}^{m} \times \mathbb{C}^{N}
$$

defined by

$$
\begin{aligned}
(x, t, z) \rightarrow( & f(x), z_{1} M_{I_{1}}(x), x_{1} z_{1} M_{I_{1}}(x), \ldots, x_{n} z_{1} M_{I_{1}}(x), \\
& \left.\ldots, z_{p} M_{I_{w}}(x), x_{1} z_{w} M_{I_{w}}(x), \ldots, x_{n} z_{w} M_{I_{w}}(x), t\right) .
\end{aligned}
$$

Denote $\Psi\left(\left(k_{1}, j_{1}\right), \ldots,\left(k_{w}, j_{w}\right), r\right):=\left(\psi_{1}(x, z), \ldots, \psi_{m+N}(x, z)\right)$. It is easy to see that

$$
\Gamma\left(\left(k_{1}, j_{1}\right), \ldots,\left(k_{w}, j_{w}\right), r\right)
$$

is the closure of

$$
\Psi\left(\left(k_{1}, j_{1}\right), \ldots,\left(k_{w}, j_{w}\right), q\right)\left(V\left(\left(k_{1}, j_{1}\right), \ldots,\left(k_{w}, j_{w}\right)\right), r\right) .
$$

Let $G\left(\left(k_{1}, j_{1}\right), \ldots,\left(k_{w}, j_{w}\right), r\right)=\operatorname{graph}\left(\Psi\left(\left(k_{1}, j_{1}\right), \ldots,\left(k_{w}, j_{w}\right), r\right)\right)$. A basis of the ideal $I$ of the set $G\left(\left(k_{1}, j_{1}\right), \ldots,\left(k_{w}, j_{w}\right), r\right)$ in the ring $\mathbb{C}\left[x_{1}, \ldots, x_{n}, x_{n+1}, t\right.$, $\left.z_{1}, \ldots, z_{w} ; y_{1}, \ldots, y_{m+N}\right]$ is given by the polynomials

$$
\begin{aligned}
\left\{b_{j}: j=1, \ldots, w\right\} & \cup\left\{z_{r} M_{I_{r}\left(k_{r}, j_{r}\right)}(x)-1: r=1, \ldots, s\right\} \cup\left\{t x_{r}-1\right\} \\
\cup & \left\{y_{i}-\psi_{i}(x, z): i=1, \ldots, m+N\right\} .
\end{aligned}
$$


To compute a basis $\mathcal{B}\left(\left(k_{1}, j_{1}\right), \ldots,\left(k_{w}, j_{w}\right), r\right)$ of the ideal of the $\operatorname{set} \operatorname{cl}\left(\Gamma\left(\left(k_{1}, j_{1}\right), \ldots\right.\right.$, $\left.\left(k_{w}, j_{w}\right), r\right)$, it is enough to compute a Gröbner basis $\mathcal{A}\left(\left(k_{1}, j_{1}\right), \ldots,\left(k_{w}, j_{w}\right), r\right)$ of the ideal $I$ in $\mathbb{C}[x, t, z, y]$ with respect to the lexicographic order in which $y<x, t, z$ (see, e.g., [14]) and then to take

$$
\mathcal{B}\left(\left(k_{1}, j_{1}\right), \ldots,\left(k_{w}, j_{w}\right), r\right)=\mathcal{A}\left(\left(k_{1}, j_{1}\right), \ldots,\left(k_{w}, j_{w}\right), r\right) \cap \mathbb{C}\left[y_{1}, \ldots, y_{m+N}\right] .
$$

\section{Consequently,}

$$
\begin{aligned}
K_{\infty}(f, X)=\bigcup_{\left(\left(k_{1}, j_{1}\right), \ldots,\left(k_{w}, j_{w}\right)\right), r}\left\{y \in \mathbb{C}^{m}: h(y, 0, \ldots, 0)=0\right. \\
\left.\quad \text { for every } h \in \mathcal{B}\left(\left(k_{1}, j_{1}\right), \ldots,\left(k_{w}, j_{w}\right), r\right)\right\} .
\end{aligned}
$$

The computation of the set $K_{0}(f, X)$ is standard. Let $I(X)=\left(b_{1}, \ldots, b_{q}\right)$. Consider the set

$$
U:=\left\{x \in \mathbb{C}^{n+1}: b_{j}=0, j=1, \ldots, w ; M_{I_{r}}=0 ; r=1, \ldots, s\right\}
$$

Now we can consider a morphism $f: U \rightarrow \mathbb{C}^{m}$. We have $K_{0}(f, X)=\overline{f(U)}$. Let $\Gamma$ be a graph of $\left.f\right|_{U}$ and $I=I(\Gamma)$. A basis of the ideal $I$ is given by the polynomials

$$
\left\{b_{j}: j=1, \ldots, w ;\right\} \cup\left\{M_{I_{r}}(x): r=1, \ldots, p\right\} \cup\left\{y_{i}-f_{i}: i=1, \ldots, m\right\} .
$$

To compute a basis $\mathcal{B}$ of the ideal $I$ it is enough to compute a Gröbner basis $\mathcal{A}$ of the ideal $I$ in $\mathbb{C}\left[x_{1}, \ldots, x_{n+1} ; y_{1}, \ldots, y_{m}\right]$ and then to take

$$
\mathcal{B}=\mathcal{A} \cap \mathbb{C}\left[y_{1}, \ldots, y_{m}\right]
$$

Consequently, $K_{0}(f, X)=\bigcup\left\{y \in \mathbb{C}^{m}: h(y, 0, \ldots, 0)=0\right.$ for every $\left.h \in \mathcal{B}\right\}$.

Acknowledgements We would like to thank Nguyen Xuan Viet Nhan and Nguyen Hong Duc for helpful discussion during the preparation of this paper. We would also like to thank the referees for their careful reading, valuable comments, and suggestions. S. T. Đinh is partially supported by the Vietnam National Foundation for Science and Technology Development (NAFOSTED) grant 101.04-2017.12. Z. Jelonek is partially supported by Narodowe Centrum Nauki grant no. 2015/17/B/ST1/02637.

Open Access This article is distributed under the terms of the Creative Commons Attribution 4.0 International License (http://creativecommons.org/licenses/by/4.0/), which permits unrestricted use, distribution, and reproduction in any medium, provided you give appropriate credit to the original author(s) and the source, provide a link to the Creative Commons license, and indicate if changes were made.

\section{References}

1. D’Acunto, D., Grandjean, V.: On gradient at infinity of semialgebraic functions. Ann. Pol. Math. 87, 39-49 (2005)

2. Cox, D., Little, J., O'Shea, D.: Ideals, Varieties, and Algorithms. Undergraduate Texts in Mathematics, 3rd edn. Springer, New York (2007) 
3. Ehresmann, C.: Les connexions infinitésimales dans un espace fibré différentiable. In: Colloque de Topologie (Espaces Fibrés), pp. 29-55. Masson, Paris (1950)

4. Eisenbud, D., Huneke, C., Vasconcelos, W.: Direct methods for primary decomposition. Invent. Math. 110(2), 207-235 (1992)

5. Flores, A.G., Teissier, B.: Local polar varieties in the geometric study of singularities. Ann. Fac. Sci. Toulouse Math. 27(4), 679-775 (2018)

6. Gianni, P., Trager, B., Zacharias, G.: Gröbner bases and primary decomposition of polynomial ideals. J. Symb. Comput. 6, 149-167 (1988)

7. Greuel, G.-M., Pfister, G.: A Singular Introduction to Commutative Algebra, 2nd edn. With contributions by Olaf Bachmann, Christoph Lossen, and Hans Schönemann. Springer, Berlin (2008)

8. Jelonek, Z.: On the generalized critical values of polynomial mappings. Manuscr. Math. 110(2), 145157 (2003)

9. Jelonek, Z.: On asymptotic critical values and the Rabier theorem. In: Hironaka, H., Janeczko, S., Łojasiewicz, S. (eds.) Geometric Singularity Theory. Banach Center Publication, vol. 65, pp. 125133. Polish Academy of Science, Warsaw (2004)

10. Jelonek, Z., Kurdyka, K.: Quantitative generalized Bertini-Sard theorem for smooth affine varieties. Discrete Comput. Geom. 34(4), 659-678 (2005)

11. Krick, T., Logar, A.: An algorithm for the computation of the radical of an ideal in the ring of polynomials. In: Applied Algebra, Algebraic Algorithms and Error-Correcting Codes. Lecture Notes in Computer Science, vol. 539, pp. 195-205. Springer, Berlin (1991)

12. Kurdyka, K., Orro, P., Simon, S.: Semialgebraic Sard theorem for generalized critical values. J. Differ. Geom. 56(1), 67-92 (2000)

13. Mather, J.: Notes on topological stability. Bull. Am. Math. Soc. (N.S.) 49(4), 475-506 (2012)

14. Pauer, F., Pfeifhofer, M.: The theory of Gröbner bases. Enseign. Math. 34, 215-232 (1988)

15. Rabier, P.J.: Ehresmann fibrations and Palais-Smale conditions for morphisms of Finsler manifolds. Ann. Math. 146(3), 647-691 (1997)

16. Serre, J.-P.: Géométrie algébrique et géométrie analytique. Ann. Inst. Fourier Grenoble 6, 1-42 (1956)

17. Teissier, B.: Variétés polaires. II. Multiplicités polaires, sections planes, et conditions de Whitney. In: Algebraic Geometry (La Rábida, 1981). Lecture Notes in Mathematics, vol. 961, pp. 314-491. Springer, Berlin (1982)

18. Thom, R.: Ensembles et morphismes stratifiés. Bull. Am. Math. Soc. 75, 240-284 (1969)

19. Uddin, M.S.: Computing dimension of affine varieties using Gröbner basis approach. IOSR J. Math. 8(3), 36-39 (2013)

20. Verdier, J.-L.: Stratifications de Whitney et théorème de Bertini-Sard. Invent. Math. 36, 295-312 (1976)

21. Whitney, H.: Local properties of analytic varieties. In: Cairns, S.S. (ed.) Differential and Combinatorial Topology, pp. 205-244. Princeton University Press, Princeton (1965)

22. Whitney, H.: Tangents to an analytic variety. Ann. Math. 81, 496-549 (1965)

Publisher's Note Springer Nature remains neutral with regard to jurisdictional claims in published maps and institutional affiliations. 